Article

\title{
One-Dimensional Alternating Extended Hubbard Model at Quarter-Filling and Its Applications to Structural Instabilities of Organic Conductors
}

\author{
M. Ménard and C. Bourbonnais *(iD) \\ Regroupement Québécois sur les Matériaux de Pointe, Départememt de physique, Université de Sherbrooke, \\ Sherbrooke, QC J1K 2R1, Canada; menarmar@gmail.com \\ * Correspondence: Claude.Bourbonnais@Usherbrooke.ca
}

Received: 17 September 2020; Accepted: 6 October 2020; Published: 16 October 2020

check for updates

\begin{abstract}
The one-dimensional extended Hubbard model with lattice dimerization and alternated site potentials is analyzed using the renormalization group method. The coupling of electrons to structural degrees of freedom such as the anion lattice and acoustic phonons is investigated to obtain the possible instabilities against the formation of lattice superstructures. Applications of the theory to anionic and spin-Peierls instabilities in the Fabre and Bechgaard salts series of organic conductors and ordered alloys are presented and discussed.
\end{abstract}

Keywords: interacting electrons in one dimension; electronic and lattice instabilities; renormalization group method; organic conductors

\section{Introduction}

The discovery of the amazing variety of electronic and structural phases in the Bechgaard and Fabre charge transfer salts series (TMTSF) ${ }_{2} X$ and (TMTTF $)_{2} X$ over the last four decades or so continues to arouse great interest in the field of organic conductors [1-13]. This research paper is devoted to explore some theoretical aspects about the role played by lattice commensurability in the origin and the development of these phases.

The part played by lattice commensurability was very early suspected to be among the determinant factors behind the occurence of electronic and lattice instabilities found in the phase diagram of these charge transfer salts. This is the case of the importance attributed to the weak but finite dimerization of the organic stacks. It is characterized by a lattice periodicity of wave vector $4 k_{F}$ ( $k_{F}$ being the Fermi wave vector along the stacks) that coincides with the one of the anion lattice $X[14,15]$. This superstructure superimposes to the basic quarter-filled commensurability of the hole band structure, which is fixed by the complete charge transfer and stoichiometry of the salts. This introduces half-filling type of Coulomb Umklapp scattering along the organic stacks, known to have a strong impact on low energy electron correlations in one spatial dimension (1D) [16-18]. Its magnitude was shown to be a key element in controlling the strength of magnetism and Mott insulating behavior across the whole phase diagram of both series of materials [15,19-21].

Another source of lattice periodicity for charge carriers of organic stacks comes from degrees of freedom associated to the anion $X$ lattice, whose $4 k_{F}$ periodicity is intrinsically linked to that of dimerization [15]. Non-centro-symmetrical anions like $X=\mathrm{ReO}_{4}^{-}, \mathrm{ClO}_{4}^{-}, \mathrm{BF}_{4}^{-}, \ldots$ and to a certain extend centrosymmetric ones like $\mathrm{X}=\mathrm{PF}_{6}^{-}, \mathrm{AsF}_{6}^{-} \ldots$ can order and form lattice superstructures also called anion orderings (AO) $[9,10,22-24]$. The coupling between anion degrees of freedom and charge carriers makes AO inevitably entangled to various electronic instabilities of organic stacks [10,25].

The interplay between superimposed lattice periodicities and electronic instabilities has been further revealed by chemistry. A notable example that we will focus on in the present work 
combines both families of compounds in the form of the alloys [(TMTSF $\left.)_{1-x}(\text { TMTTF })_{x}\right]_{2} \mathrm{ReO}_{4}$ with the asymmetrical anion $\mathrm{ReO}_{4}^{-}$. Remarkably, in the case where $\mathrm{x} \sim 0.5$, the alloy is found to be ordered [26]. The alternation of different organic molecules along the stacks then acts as a distinct site potential with the same $4 k_{F}$ periodicity found for the bond centered dimerization [26-29]. Transport and structural studies have showed that these superimposed types of commensurability have a strong impact on both the Mott insulating state and the staggered $\mathrm{ReO}_{4}^{-} \mathrm{AO}$ found in the pure limits [26].

An alternating site periodicity can also be generated dynamically in pure systems of the (TMTTF $)_{2} X$ salts. This occurs for the charge ordering (CO) phase transition [30-32], which introduces a charge disproportionation in the dimerized unit cell that breaks the inversion symmetry and gives rise to a ferroelectric state [29]. The importance of Coulomb interaction between carriers of different molecular units in the unit cell has pointed to the key role of underlying quarter-filling commensurability component of Umklapp scattering in the electronic origin of this phase [33-36].

The existence of a CO state in the (TMTTF) ${ }_{2} \mathrm{X}$ series has been found to interfere with the formation of another ordered state involving both spin and lattice degrees of freedom. This the case of the spin-Peierls (SP) instability which corresponds to a $2 k_{F}$ bond like tetramerization of the organic stacks that opens a gap in the spin sector $[37,38]$. On experimental grounds [39], the presence of the CO state is found to particularly weaken the strength of the SP order, an effect as we will see that results from the interplay between different types of commensurability [35,40-43].

In the present paper we shall address theoretically the combined influence of different types of lattice commensurability in the framework of a generalized extended Hubbard model. This will be examined in the $1 \mathrm{D}$ regime that characterizes both series of materials at relatively high temperature. The combined influence of site and bond dimerization lattice potentials together with the respective modulation of Coulomb interaction terms are considered. Their impact on excitation gaps and singular correlations of the phase diagram is analyzed using a two-loop renormalization group (RG) method in the electron gas limit. The $2 k_{F}$ bond and site density-wave correlations with their phase relation to the underlying alternated lattice are determined. The model is integrated to the one proposed by Riera and Poilblanc for the mechanism of anion ordering in the (TMTSF) ${ }_{2} X$ and (TMTTF) ${ }_{2} X$ salts. The coupling of anions displacement to charge carriers in systems like the alloys [(TMTSF $\left.)_{1-x}(\mathrm{TMTTF})_{x}\right]_{2} \mathrm{ReO}_{4}$ is derived and the reduction (magnification) of the staggered anion ordering (Mott temperature scale) is obtained and shown to compare favorably with previous experiments performed on these materials [26]. Finally, the model is also applied to the influence of $\mathrm{CO}$ state on the pressure profile of the spin-Peierls instability occurring in (TMTTF $)_{2} X$ with centrosymmetric anions. By modeling $\mathrm{CO}$ in terms of a site lattice potential, it is found that lattice coupling responsible for the SP tetramerization is strongly altered by the presence of $\mathrm{CO}$ which mainly governs the variation of the temperature scale of the SP instability observed under pressure.

\section{Alternating Extended Hubbard Model}

\section{The Model and Its Continuum Limit}

We consider a 1D model of interacting electrons on a bipartite lattice. The Hamiltonian $H=H_{0}+H_{I}$ consists of the one-body term

$$
\begin{gathered}
H_{0}=-\sum_{r, \sigma}\left[(t+\delta t) a_{r, \sigma}^{\dagger} b_{r, \sigma}+(t-\delta t) a_{r, \sigma}^{\dagger} b_{r-1, \sigma}+\text { H.c. }\right] \\
+\epsilon_{0} \sum_{r, \sigma}\left(m_{r, \sigma}-n_{r, \sigma}\right)
\end{gathered}
$$


and the interaction term,

$$
\begin{aligned}
H_{I} & =\frac{1}{2} \sum_{r, \sigma}\left[(U+\delta U) m_{r, \sigma} m_{r,-\sigma}+(U-\delta U) n_{r, \sigma} n_{r,-\sigma}\right] \\
& +\sum_{r, \sigma_{1,2}}(V+\delta V) m_{r, \sigma_{1}} n_{r, \sigma_{2}}+(V-\delta V) m_{r, \sigma_{1}} n_{r-1, \sigma_{2}} .
\end{aligned}
$$

Here, $a_{r \sigma}^{(+)}$and $b_{r \sigma}^{(+)}$are the annihilation (creation) operators of electrons of spin $\sigma$ at even and odd sites of a bipartite lattice; $m_{r \sigma}=a_{r \sigma}^{\dagger} a_{r \sigma}$ and $n_{r \sigma}=b_{r \sigma}^{\dagger} b_{r \sigma}$ stand for the number operators on the corresponding sites. At quarter-filling, we have $\left\langle m_{r \sigma}\right\rangle=\left\langle n_{r \sigma}\right\rangle=\frac{1}{2}$. The standard extended Hubbard part of $H$ is described by the hopping integral $t$, and the intra- and inter-site interaction terms $U$ and $V$. The model includes the influence of two different alternating potentials. We have first a small lattice dimerization which modulates the hopping integral by $\delta t[14,15]$ and the nearest-neighbor interaction $V$ by a corresponding positive $\delta V$, whether the electrons interact on shorter or longer bonds as expected from quantum chemistry calculations on dimerized chains materials like the Fabre and the Bechgaard salts [44]. The second potential to be examined is an alternating site potential of amplitude $\epsilon_{0}$ [27]. The latter can be found in practice in quasi-1D organic ordered alloys in which the molecular species alternates from site to site [26], or as a result of charge ordering which in systems like the Fabre salts introduces a charge disproportionation modulated along the stacks [31,32]. Besides the modulation of site energy, a molecular alternation is expected to modify Coulomb integral $U$ from site to site, which is taken into account by adding (substracting) a negative $\delta U$ when the site energy is increased (reduced) by $\epsilon_{0}$ (see Figure 1).

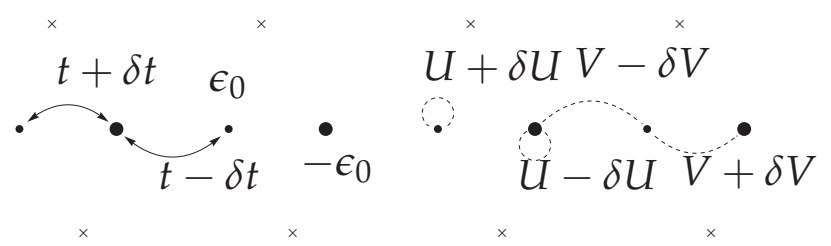

Figure 1. One dimensional alternating extended Hubbard model. The small and big full circles depict higher and lower potential energies, and crosses refer to the positions of anions in systems like $(\mathrm{TMTSF})_{2} \mathrm{X},(\mathrm{TMTTF})_{2} \mathrm{X}$ and their alloys.

Using the Fourier transforms of each sublattice operator,

$$
\begin{aligned}
& a_{r, \sigma}=\sqrt{\frac{2}{N}} \sum_{k} a_{k \sigma} e^{i k r}, \\
& b_{r, \sigma}=\sqrt{\frac{2}{N}} \sum_{k} b_{k \sigma} e^{i k r},
\end{aligned}
$$

the one-body part of the Hamiltonian is diagonalized in the form

$$
H_{0}=-\sum_{k, \sigma} E_{k} d_{k, \sigma}^{+} d_{k, \sigma}+\sum_{k, \sigma} E_{k} f_{k, \sigma}^{+} f_{k, \sigma}
$$

which is written in terms of the new operators $d_{k, \sigma}^{(+)}$and $f_{k, \sigma}^{(+)}$for the lower and upper bands following the transformations

$$
\begin{aligned}
& a_{k, \sigma}^{\dagger}=e^{-i\left(\frac{k}{4}-\frac{v_{k}}{2}\right)}\left(\sin \frac{\gamma_{k}}{2} d_{k, \sigma}^{\dagger}+\cos \frac{\gamma_{k}}{2} f_{k, \sigma}^{\dagger}\right), \\
& b_{k, \sigma}^{+}=e^{i\left(\frac{k}{4}-\frac{v_{k}}{2}\right)}\left(\cos \frac{\gamma_{k}}{2} d_{k, \sigma}^{\dagger}-\sin \frac{\gamma_{k}}{2} f_{k, \sigma}^{+}\right),
\end{aligned}
$$


where the phase factors obey the relations

$$
\begin{aligned}
\epsilon_{0} & =E_{k} \cos \gamma_{k}, \\
2 t \cos \frac{k}{2} & =E_{k} \sin \gamma_{k} \cos v_{k}, \\
2 \delta t \sin \frac{k}{2} & =E_{k} \sin \gamma_{k} \sin v_{k} .
\end{aligned}
$$

The amplitude of the spectrum for each band is given by

$$
E_{k}=2 \sqrt{t^{2} \cos ^{2} \frac{k}{2}+\delta t^{2} \sin ^{2} \frac{k}{2}+\left(\frac{\epsilon_{0}}{2}\right)^{2}}
$$

At quarter filling the lower band is occupied up to the Fermi points $\pm k_{F}= \pm \pi / 2$, corresponding to the Fermi level $-E_{k_{F}} \equiv-E_{F}$ where

$$
E_{F}=\sqrt{2 t^{2}+2 \delta t^{2}+\epsilon_{0}^{2}}
$$

is the Fermi energy. The lower and upper bands in Figure 2 are separated by a gap $\Delta=2 E_{\pi}=$ $2 \sqrt{4 \delta t^{2}+\epsilon_{0}^{2}}$.

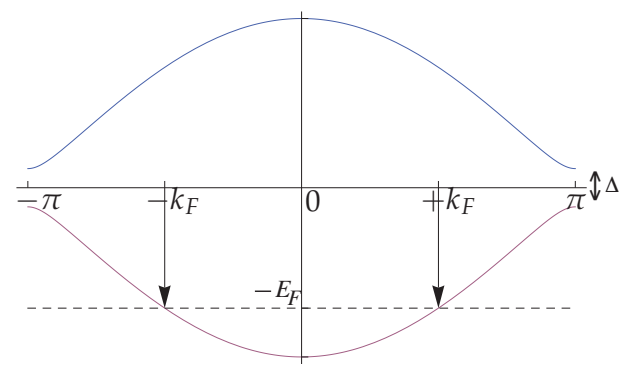

Figure 2. Electron spectrum of the one-dimensional extended alternated Hubbard model.

For the interacting part of the Hamiltonian, we shall focus on the weak coupling case by considering only low energy scattering processes taking place within the lower ' $d$ ' band (perturbative corrections coming from the upper $f$ band at higher energy have been considered by Penc and Mila [27]). This yields

$$
\begin{aligned}
H_{I}=\frac{1}{2 N} \sum_{\{k, \sigma\}} g\left(k_{1}, k_{2}, k_{3}, k_{4}\right) \delta_{k_{4}+k_{3}, k_{1}+k_{2}+G} \\
\times d_{k_{4}, \sigma_{4}}^{+} d_{k_{3}, \sigma_{3}}^{+} d_{k_{2}, \sigma_{2}} d_{k_{1}, \sigma_{1}}+\ldots
\end{aligned}
$$

where $G=0(G= \pm 2 \pi)$ stands for normal (Umklapp) scattering processes. The interaction amplitude reads

$$
\begin{aligned}
g\left(k_{1}, k_{2}, k_{3}, k_{4}\right)= & (U+\delta U) e^{i\left(2 v_{[3]}-\frac{G}{4}\right)} \prod_{i=1}^{4} \sin \frac{\gamma_{k_{i}}}{2} \\
& +(U-\delta U) e^{-i\left(2 v_{[3]}-\frac{G}{4}\right)} \prod_{i=1}^{4} \cos \frac{\gamma_{k_{i}}}{2} \\
& +\left(2 V \cos \frac{k_{4}-k_{1}}{2}-2 i \delta V \sin \frac{k_{4}-k_{1}}{2}\right) e^{i\left(\frac{G}{4}+2 v_{[1]}\right)} \sin \frac{\gamma_{k_{4}}}{2} \cos \frac{\gamma_{k_{3}}}{2} \cos \frac{\gamma_{k_{2}}}{2} \sin \frac{\gamma_{k_{1}}}{2} \\
& +\left(2 V \cos \frac{k_{4}-k_{1}}{2}+2 i \delta V \sin \frac{k_{4}-k_{1}}{2}\right) e^{-i\left(\frac{G}{4}+2 v_{[1]}\right)} \cos \frac{\gamma_{k_{4}}}{2} \sin \frac{\gamma_{k_{3}}}{2} \sin \frac{\gamma_{k_{2}}}{2} \cos \frac{\gamma_{k_{1}}}{2}
\end{aligned}
$$


where

$$
v_{[1,3]}=\frac{v_{k_{4}} \mp v_{k_{3}} \pm v_{k_{2}}-v_{k_{1}}}{4} .
$$

In weak coupling, the lattice model can be transposed into the continuum limit of the electron gas model. This standard procedure first consists in the linearization of the spectrum around the Fermi points $p k_{F}= \pm k_{F}$

$$
E_{F}-E_{k} \approx E_{p}(k)=v_{F}\left(p k-k_{F}\right)
$$

where

$$
v_{F}=\frac{t^{2}-\delta t^{2}}{\sqrt{2 t^{2}+2 \delta t^{2}+\epsilon_{0}^{2}}}
$$

is the Fermi velocity ( $\hbar=1$ throughout). The operators for left and right moving electrons become $d_{ \pm, k, \sigma}\left(d_{ \pm, k, \sigma}^{\dagger}\right)$. The linearized spectrum is limited by an energy cut-off $E_{0} / 2=E_{F}$ on either side of the Fermi level, where $E_{0}$ is the total width of the lower band. The interaction is defined with respect to the Fermi points giving rise to four possible coupling constants, commonly noted as $g_{i=1-4}[16,17,45]$. Together with the one-electron part, this leads to the usual form for the Hamiltonian of the electron gas model. For the lower band, one gets

$$
\begin{aligned}
& H=\sum_{k, p, \sigma} E_{p}(k) d_{p, k \sigma}^{\dagger} d_{p, k \sigma}+\frac{1}{N} \sum_{\{k, q, \sigma\}} g_{1} d_{+, k_{1}+q, \sigma_{1}}^{+} d_{-, k_{2}-q, \sigma_{2}}^{\dagger} d_{+, k_{2}, \sigma_{2}} d_{-, k_{1}, \sigma_{1}} \\
&+g_{2} d_{+, k_{1}+q, \sigma_{1}}^{+} d_{-, k_{2}-q, \sigma_{2}}^{+} d_{-, k_{2}, \sigma_{2}} d_{+, k_{1}, \sigma_{1}} \\
&+ \frac{1}{2}\left(g_{3}^{+} d_{+, k_{1}+q, \sigma_{1}}^{\dagger} d_{+, k_{2}-q+G_{,} \sigma_{2}}^{\dagger} d_{-, k_{2}, \sigma_{2}} d_{-, k_{1}, \sigma_{1}}+\text { H.c. }\right) \\
&+ \frac{1}{2} g_{4} d_{+, k_{1}+q, \sigma_{1}}^{+} d_{+, k_{2}-q, \sigma_{2}}^{+} d_{+, k_{2}, \sigma_{2}} d_{+, k_{1}, \sigma_{1}} \\
&+\frac{1}{2} g_{4} d_{-, k_{1}+q, \sigma_{1}}^{+} d_{-, k_{2}-q, \sigma_{2}}^{+} d_{-, k_{2}, \sigma_{2}} d_{-, k_{1}, \sigma_{1}}
\end{aligned}
$$

where the scattering amplitudes in the standard terminology [16,17], are $g_{1} \equiv g\left(+k_{F},-k_{F},+k_{F},-k_{F}\right)$, $g_{2} \equiv g\left(+k_{F},-k_{F},-k_{F},+k_{F}\right), g_{3}^{ \pm} \equiv g\left( \pm k_{F}, \pm k_{F}, \mp k_{F}, \mp k_{F}\right)$ and $g_{4} \equiv g\left( \pm k_{F}, \pm k_{F}, \pm k_{F}, \pm k_{F}\right)$, which in order stand for backward, forward, half-filling Umklapp, and inner branch forward scattering processes. According to the expression (14) for the lattice model, the bare $g_{i}$ amplitudes are given by

$$
\begin{aligned}
g_{1} & =U\left(1+\epsilon_{0}^{2} / E_{F}^{2}\right)-2 \delta U \epsilon_{0} / E_{F}+4 t \delta V \delta t / E_{F}^{2} \\
g_{2} & =U\left(1+\epsilon_{0}^{2} / E_{F}^{2}\right)-2 \delta U \epsilon_{0} / E_{F}+2 V\left(1-\epsilon_{0}^{2} / E_{F}^{2}\right) \\
g_{3}^{p} & =\left[U\left(1+\epsilon_{0}^{2} / E_{F}^{2}\right)-2 \delta U \epsilon_{0} / E_{F}\right] \frac{2 t \delta t}{t^{2}+\delta t^{2}} \\
& +2 \delta V\left(1-\epsilon_{0}^{2} / E_{F}^{2}\right) \\
& +i p\left[2 U \epsilon_{0} / E_{F}-\delta U\left(1+\epsilon_{0}^{2} / E_{F}^{2}\right)\right] \frac{t^{2}-\delta t^{2}}{t^{2}+\delta t^{2}} \\
& \equiv\left|g_{3}\right| e^{i p \theta} \\
g_{4} & =g_{2} .
\end{aligned}
$$

From these expressions, we first observe that alongside the bond alternation of hopping $\delta t$ and the modulation of on-site energy $\epsilon_{0}$, both local $(\delta U<0)$ and intersite $(\delta V>0)$ modulations of interactions contribute to an increase of backscattering and half-filling Umklapp. Furthermore, the local components of the modulation contribute to an imaginary part of $g_{3}^{p}=\left|g_{3}\right| e^{i p \theta}$, Ref. [28] whose argument $\theta$ plays an important role for the relative phase of charge/spin density-wave correlations with respect to the lattice. This will be analyzed in Section 3.2. 


\section{Renormalization Group Results}

\subsection{Formulation and Coupling Constants}

We apply the renormalization group approach to our effective electron gas model described above. In the following, we sketch out only the main steps of the procedure that will be useful later on for applications $[17,46,47]$. We follow Ref. [46] and write the partition function in the functional integral form,

$$
Z=\iint \mathfrak{D} \psi^{*} \mathfrak{D} \psi e^{S\left[\psi^{*}, \psi\right]},
$$

over a set anticommuting fermion variables $\left\{\psi^{*}, \psi\right\}$, where the measure is

$$
\mathfrak{D} \psi^{*} \mathfrak{D} \psi=\prod_{\bar{k}, p, \sigma} d \psi_{p, \sigma}^{*}(\bar{k}) d \psi_{p, \sigma}(\bar{k})
$$

and $\bar{k}=\left(k, \omega_{n}=(2 n+1) \pi T\right) \quad\left(k_{B}=1 \quad\right.$ throughout $) . \quad$ The action $S=S_{0}+S_{I}$ splits into a free-quadratic- $\left(S_{0}\right)$ and an interacting-quartic- $\left(S_{I}\right)$ parts,

$$
\begin{aligned}
& S\left[\psi^{*}, \psi\right]=\sum_{\bar{k}, p, \sigma} z^{-1}\left[G_{p}^{0}(\bar{k})\right]^{-1} \psi_{p, \sigma}^{*}(\bar{k}) \psi_{p, \sigma}(\bar{k}) \\
& \quad-\frac{T}{N} \sum_{\bar{k}_{1}, \bar{k}_{1}^{\prime}, \bar{k}_{2}, \bar{k}_{2}^{\prime}} \sum_{\sigma, \sigma^{\prime}} \delta_{\bar{k}_{1}+\bar{k}_{2}, \bar{k}_{1}^{\prime}+\bar{k}_{2}^{\prime}( \pm \bar{G})} \\
& \quad \times\left\{z_{1} g_{1} \psi_{+, \sigma}^{*}\left(\bar{k}_{1}^{\prime}\right) \psi_{-, \sigma^{\prime}}^{*}\left(\bar{k}_{2}^{\prime}\right) \psi_{+, \sigma^{\prime}}\left(\bar{k}_{2}\right) \psi_{-, \sigma}\left(\bar{k}_{1}\right)\right. \\
& \quad+z_{2} g_{2} \psi_{+, \sigma}^{*}\left(\bar{k}_{1}^{\prime}\right) \psi_{-, \sigma^{\prime}}^{*}\left(\bar{k}_{2}^{\prime}\right) \psi_{-, \sigma^{\prime}}\left(\bar{k}_{2}\right) \psi_{+, \sigma}\left(\bar{k}_{1}\right) \\
& \quad+\frac{1}{2} z_{3}\left[g_{3}^{+} \psi_{+, \sigma}^{*}\left(\bar{k}_{1}^{\prime}\right) \psi_{+, \sigma^{\prime}}^{*}\left(\bar{k}_{2}^{\prime}\right) \psi_{-, \sigma^{\prime}}\left(\bar{k}_{2}\right) \psi_{-, \sigma}\left(\bar{k}_{1}\right)+\right.\text { “c.c.”] } \\
& \quad+\frac{1}{2} g_{4} \psi_{+, \sigma}^{*}\left(\bar{k}_{1}^{\prime}\right) \psi_{+, \sigma^{\prime}}^{*}\left(\bar{k}_{2}^{\prime}\right) \psi_{+, \sigma^{\prime}}\left(\bar{k}_{2}\right) \psi_{+, \sigma}\left(\bar{k}_{1}\right) \\
& \left.\quad+\frac{1}{2} g_{4} \psi_{-, \sigma}^{*}\left(\bar{k}_{1}^{\prime}\right) \psi_{-, \sigma^{\prime}}^{*}\left(\bar{k}_{2}^{\prime}\right) \psi_{-, \sigma^{\prime}}\left(\bar{k}_{2}\right) \psi_{-, \sigma}\left(\bar{k}_{1}\right)\right\},
\end{aligned}
$$

where $z$ and $z_{1,2,3}$ are in order the renormalization factors for the one-particle propagator

$$
G_{p}^{0}(\bar{k})=\left[i \omega_{n}-E_{p}(k)\right]^{-1}
$$

and the four-points electron-electron vertices $\Gamma_{1,2,3}$. The $z_{i}$ are combined to $z$ to give the renormalization factors $z^{2} z_{i}$ for each coupling $g_{i=1,2,3}$. At the bare level, the couplings $g_{i}$ are defined at the band edge energy cutoff $\Lambda_{0}\left(\equiv E_{F}\right)$ above and below the Fermi level where both $z$ and the $z_{i}^{\prime}$ 's equal unity.

The RG transformation is standard and consists in the succesive integrations of electronic degrees of freedom, denoted by $\bar{\psi}^{(*)}$, in outer energy shell of thickness $\Lambda(\ell) d \ell$ on both sides of the Femi level of the lower band, where $\Lambda(\ell)=\Lambda_{0} e^{-\ell}$ is the cutoff at the step $\ell$ of the RG procedure. The integration of degrees of freedom from step $\ell$ to $\ell+d \ell$ is achieved perturbatively. This recursive transformation can be written in the form

$$
\begin{aligned}
& Z \sim \iint\left[\mathfrak{D} \psi^{*} \mathfrak{D} \psi\right]_{\ell+d \ell} e^{S\left[\psi^{*}, \psi\right]_{\ell}} \iint\left[\mathfrak{D} \bar{\psi}^{*} \mathfrak{D} \bar{\psi}\right]_{d \ell} e^{S_{0}\left[\bar{\psi}^{*}, \bar{\psi}\right]_{d \ell}} \times e^{S_{I}\left[\psi^{*}, \psi, \bar{\psi}^{*}, \bar{\psi}\right]_{d \ell}} \\
& \sim \iint\left[\mathfrak{D} \psi^{*} \mathfrak{D} \psi\right]_{\ell+d \ell} e^{S\left[\psi^{*}, \psi\right]_{\ell}} e^{\sum_{n} \frac{1}{n !}\left\langle S_{I}^{n}\left[\psi^{*}, \psi, \bar{\psi}^{*}, \bar{\psi}\right]_{d \ell}\right\rangle_{0, d \ell}} \\
& \propto \iint\left[\mathfrak{D} \psi^{*} \mathfrak{D} \psi\right]_{\ell+d \ell} e^{S\left[\psi^{*}, \psi\right]_{\ell+d \ell},}
\end{aligned}
$$


where $\left\langle S_{I}^{n}\right\rangle_{0, d \ell}$ are outer shell free (loop) averages with external fermion legs in the inner energy shells at $\Lambda \leq \Lambda(\ell+d \ell)$. The effective-renormalized-action $S\left[\psi^{*}, \psi\right]_{\ell+d \ell}$ at $\ell+d \ell$ leads to the recursion transformation for the $z^{\prime} s$.

Thus for the one-particle propagator, $z(\ell+d \ell)=z(\ell) z(d \ell)$, which leads to the familiar result at the two-loop level [17,46,47],

$$
\frac{d \ln z}{d \ell}=-\frac{1}{16}\left(\pi v_{F}\right)^{-2}\left[\left(2 g_{2}-g_{1}\right)^{2}+3 g_{1}^{2}+2 g_{3}^{+} g_{3}^{-}\right],
$$

which is independent of the phase of the Umklapp term. The recursion relations $g_{i}(\ell+d \ell)=z_{i}(d \ell) z^{2}(d \ell) g_{i}(\ell)$ for the coupling constants lead to the two-loop flow equations

$$
\begin{aligned}
\frac{d}{d \ell} \bar{g}_{1} & =-\bar{g}_{1}^{2}-\frac{1}{2} \bar{g}_{1}^{3} \\
\frac{d}{d \ell}\left(2 \bar{g}_{2}-\bar{g}_{1}\right) & =\bar{g}_{3}^{+} \bar{g}_{3}^{-}-\frac{1}{2} \bar{g}_{3}^{+} \bar{g}_{3}^{-}\left(2 \bar{g}_{2}-\bar{g}_{1}\right), \\
\frac{d}{d \ell} \bar{g}_{3}^{p} & =\bar{g}_{3}^{p}\left(2 \bar{g}_{2}-\bar{g}_{1}\right) \\
& -\frac{1}{4} \bar{g}_{3}^{p}\left[\left(2 \bar{g}_{2}-\bar{g}_{1}\right)^{2}+\bar{g}_{3}^{+} \bar{g}_{3}^{-}\right], \\
\frac{d \theta}{d \ell} & =0 .
\end{aligned}
$$

The first equation for $\bar{g}_{1}$ is connected to spin degrees of freedom and is decoupled from $\left(2 \bar{g}_{2}-\bar{g}_{1}, \bar{g}_{3}^{p}\right)$, which is connected to the charge. These extend the known flow equations of the electron gas model $[17,46,47]$ to the case of a complex $g_{3}^{p}$. Note that only the amplitude of Umklapp $\left|g_{3}\right|$ renormalizes, whereas its phase $\theta$ remains scale invariant $\left[\operatorname{Im}\left(d_{\ell} \ln g_{3}^{p}\right)=0\right]$ and is then fixed at the bare level by the expression (21). The renormalization of $g_{4}$ is here neglected. However the influence of this coupling has been incorporated through the normalization $\bar{g}_{1}=g_{1} / \pi v_{\sigma}$ and $\left(2 \bar{g}_{2}-\bar{g}_{1}, \bar{g}_{3}^{p}\right)$ $=\left(2 g_{2}-g_{1}, g_{3}^{p}\right) / \pi v_{\rho}$ for the decoupled spin $(\sigma)$ and charge $(\rho)$ interactions, respectively [17], where $v_{\sigma, \rho}=v_{F} \mp g_{4} / 2 \pi$ are the spin and charge velocities.

The properties of the above flow equations are standard [17,47] and can be summarized as follows. In the spin sector for instance, the negative (positive) sign of $g_{1}$ determines the conditions for the flow to strong (weak) attractive coupling, $\bar{g}_{1}^{*} \rightarrow-2\left(\bar{g}_{1}^{*} \rightarrow 0\right)$, as $\ell \rightarrow \infty$. In the attractive case, this indicates the emergence of a spin gap $\Delta_{\sigma}$, whose scale is of the order of the cutoff energy $2 \Lambda\left(\ell_{\sigma}\right)$ at which the flow of $\bar{g}_{1}$ in (28) becomes singular at the one-loop $\mathcal{O}\left(\bar{g}_{1}^{2}\right)$ level, namely $\Delta_{\sigma} \sim 2 E_{F} e^{-1 /\left|\bar{g}_{1}\right|}$.

If we now consider the charge sector, the magnitude of $2 \bar{g}_{2}-\bar{g}_{1}$ with respect to $\left|\bar{g}_{3}\right|$ at the bare level determines the conditions for strong coupling or a charge gap $\Delta_{\rho}$. Thus for $\bar{g}_{1}-2 \bar{g}_{2} \geq\left|\bar{g}_{3}\right|$, charge degrees of freedom remain gapless since $\left|\bar{g}_{3}^{*}\right| \rightarrow 0$ and $\bar{g}_{1}^{*}-2 \bar{g}_{2}^{*}$ is non universal as $\ell \rightarrow \infty$. In the whole region where $\bar{g}_{1}-2 \bar{g}_{2}<\left|\bar{g}_{3}\right|$, both $2 \bar{g}_{2}^{*}-\bar{g}_{1}^{*} \rightarrow 2$ and $\left|\bar{g}_{3}^{*}\right| \rightarrow 2$ are marginally relevant and scale to strong coupling when $\ell \rightarrow \infty$. An order of magnitude for the charge gap $\Delta_{\rho}$ can be readily given by the singularities encountered at a finite $\ell_{\rho}$ in (29) and (30) at the one-loop, $\mathcal{O}\left(\bar{g}^{2}\right)$, level [16]. For $-\left|\bar{g}_{3}\right|<\bar{g}_{1}-2 \bar{g}_{2}<\left|\bar{g}_{3}\right|$, one has $\Delta_{\rho} \sim 2 \Lambda\left(\ell_{\rho}\right)=2 E_{F} \exp \left[-c / \sqrt{\left|\bar{g}_{3}\right|^{2}-\left(2 \bar{g}_{2}-\bar{g}_{1}\right)^{2}}\right]$, where $c=\arccos \left[\left(2 \bar{g}_{2}-\bar{g}_{1}\right) /\left|\bar{g}_{3}\right|\right]$; for $\bar{g}_{1}-2 \bar{g}_{2}=-\left|\bar{g}_{3}\right|, \Delta_{\rho}=2 E_{F} \exp \left[-\left(1 /\left|\overline{g_{3}}\right|\right)\right]$; and finally for $\bar{g}_{1}-2 \bar{g}_{2}<-\left|\bar{g}_{3}\right|$, one has $\Delta_{\rho}=2 E_{F} \exp \left[-c^{\prime} / \sqrt{\left(2 \bar{g}_{2}-\bar{g}_{1}\right)^{2}-\left|\bar{g}_{3}\right|^{2}}\right]$, where $c^{\prime}=\cosh ^{-1}\left[\left(2 \bar{g}_{2}-\bar{g}_{1}\right) /\left|\bar{g}_{3}\right|\right]$.

We display in Figure 3 the contour plot of the scale for the charge gap $e^{-\ell_{\rho}}=\Delta_{\rho} / 2 E_{F}$ at the one-loop level in the $\left(\epsilon_{0}, \delta t\right)$ plane of alternating potentials and for repulsive $(U, V)$ interactions and smaller modulations $(\delta U, \delta V)$. In the first quadrant where both $\delta t$ and $\epsilon_{0}$ are positive, the variation of $\Delta_{\rho}$ is not monotonous; it first increases with $\delta t$ and $\epsilon_{0}$ and then undergoes a smooth decreases. According to (21), both the real and imaginary parts of Umklapp increase at relatively small $\delta t$ and $\epsilon_{0}$; this is responsible for the increase of $\Delta_{\rho}$. At sufficiently large $\delta t$, however, a reduction of the imaginary 
part of $g_{3}^{p}$ becomes apparent and leads to the decrease of $\Delta_{\rho}$. A similar variation of charge gap has been obtained in the bosonization approach to the alternating Hubbard model with positive $U$ [28].

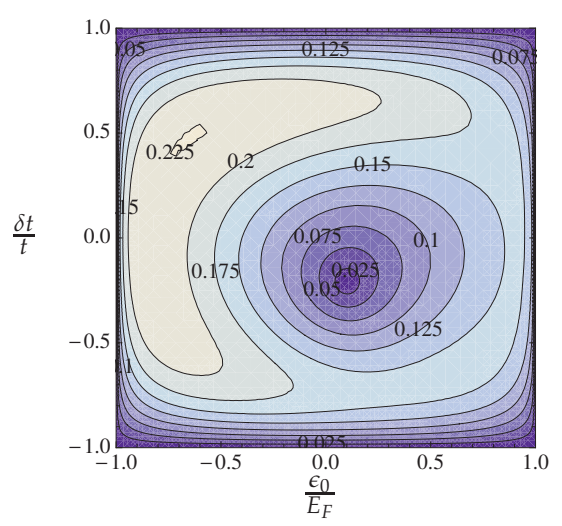

Figure 3. Contour plot of the normalized one-loop charge gap $\Delta_{\rho} / 2 E_{F}\left(\equiv e^{-\ell_{\rho}}\right)$ as a function of the alternating site $\left(\epsilon_{0}\right)$ and bond $(\delta t)$ potentials. The calculations are done for repulsive interactions $U / t=V / t=0.5$ and $\delta U / t=\delta V / t=0.1$.

Interestingly, if we broaden the analysis situation where positive $\delta U>0$ and $\delta V>0$ are considered, the competition between a positive $\epsilon_{0}$ and negative $\delta t$ can bring both the real and imaginary parts of $g_{3}^{p}$ and in turn $\Delta_{\rho}$ to zero, as shown in the second quadrant of Figure 3. Around this point, the behavior of Equations (29) and (30), as $\ell \rightarrow \ell_{\rho}$ at the one-loop level shows that the gap vanishes following the power law $\Delta_{\rho} / 2 E_{F} \sim\left[\frac{1}{2}\left|\bar{g}_{3}\right| /\left(2 \bar{g}_{2}-\bar{g}_{1}\right)\right]^{1 /\left(2 \bar{g}_{2}-\bar{g}_{1}\right)}$.

\subsection{Response Functions and Phase Diagram}

In order to analyze the nature of correlations and the possible phases of the above model, we proceed to the calculation of susceptibilities. To do so, we follow Ref. [46] and add to the action a linear coupling to a set of infinitesimal source fields $\{h\}$ to fermion pair fields. These are associated to susceptibilities that can become singular in the $2 k_{F}$ density-wave and superconducting channels. As infinitesimal terms, they can be combined at the bare level to the interaction term $S_{I}$ and treated as a perturbation. The action becomes

$$
S\left[\psi^{*}, \psi,\{h\}\right]=S_{0}\left[\psi^{*}, \psi\right]+S_{I}\left[\psi^{*}, \psi\right]+S_{h}\left[\psi^{*}, \psi,\{h\}\right],
$$

where

$$
\begin{aligned}
S_{h}\left[\psi^{*}, \psi,\{h\}\right] & =\sum_{\bar{q}}\left[\sum_{\mu} z_{\mu}^{\mathrm{s}} h_{\mu}^{\mathrm{s} *}(\bar{q}) \Delta_{\mu}(\bar{q})\right. \\
& +\sum_{\mu= \pm} z_{\theta_{\mu}}^{\mathrm{c}} h_{\mu}^{\mathrm{c} *}(\bar{q}) O_{\theta_{\mu}}(\bar{q}) \\
& +\sum_{\mu= \pm} z_{\theta_{\mu}}^{\sigma} \vec{h}_{\mu}^{\sigma *}(\bar{q}) \cdot \vec{S}_{\theta_{\mu}}(\bar{q})+\text { “c.c.”] }
\end{aligned}
$$

In the superconducting channel, the pair fields are

$$
\Delta_{\mu}(\bar{q})=\sqrt{\frac{T}{N}} \sum_{\bar{k}, \alpha, \beta} \alpha \psi_{-,-\alpha}(-\bar{k}) \sigma_{\mu}^{\alpha \beta} \psi_{+, \beta}(\bar{k}+\bar{q})
$$

for $\mu=0$ singlet (SS) and $\mu=1,2,3$ triplet (TS) superconductivity. Here $\sigma_{0}=\mathbf{1}, \sigma_{1,2,3}$ are the Pauli matrices, and $\bar{q}=\left(q, \omega_{m}=2 \pi m T\right)$. The initial pair renormalization factors at $\ell=0$ are $z_{\mu}^{\mathrm{s}}=1$. 
In the $2 k_{F}$ density-wave channel, the presence of a complex Umklapp interaction term $g_{3}^{p}$ in (21), which can be written as $g_{3}^{ \pm}= \pm\left|g_{3}\right| e^{i \theta_{ \pm}}$, where $\theta_{+}=\theta$ and $\theta_{-}=\theta-\pi$, introduces spin and charge density-wave correlations with a particular phase relation with respect to the lattice. For $2 k_{F}$ charge-density-wave (CDW), the pair field can be written in terms of two independent stationary waves,

$$
O_{\theta_{ \pm}}(\bar{q})=e^{i \theta_{ \pm}} O^{*}(\bar{q})+O(\bar{q})
$$

where

$$
O(\bar{q})=\sqrt{\frac{T}{N}} \sum_{\bar{k}, \alpha} \psi_{-, \alpha}^{*}(\bar{k}-\bar{q}) \psi_{+, \alpha}(\bar{k})
$$

for $\bar{q}=\left(q, \omega_{m}\right)$. The phase relation of $\mathrm{CDW}_{\theta_{ \pm}}$maxima and minima with respect to the lattice is shown in Figure 4 at $q=2 k_{F}$. In the absence of $\epsilon_{0}$ and $\delta U$, for instance, the imaginary part of $g_{3}^{p}$ vanishes and $\theta_{-}=\pi$ and $\mathrm{CDW}_{\theta_{-}}$correlations are centered on bonds between dimers, whereas $\theta_{+}=0$ refers to $\mathrm{CDW}_{\theta_{+}}$whose maxima are centered on dimers. In the presence of a finite site potential $\epsilon_{0}$ and/or $\delta U$, the inversion symmetry within the dimers is broken and the position of maxima for $\mathrm{CDW}_{\theta_{ \pm}}$move accordingly (see Figure 4).
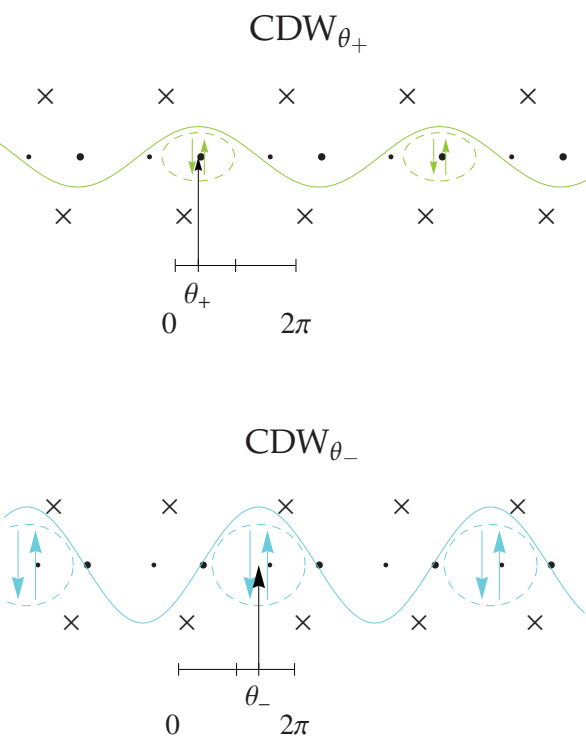

Figure 4. Site $(+)$ and bond $(-) 2 k_{F}$ charge-density-wave and their respective phase $\left(\theta_{ \pm}\right)$relative to the alternated lattice.

A similar decomposition can be made for $2 k_{F}$ spin-density-wave $\left(\mathrm{SDW}_{\theta_{ \pm}}\right)$by introducing

$$
\vec{S}_{\theta_{ \pm}}(\bar{q})=e^{i \theta_{ \pm}} \vec{S}^{*}(\bar{q})+\vec{S}(\bar{q})
$$

for $\mathrm{SDW}_{\theta_{ \pm}}$, where

$$
\vec{S}(\bar{q})=\sqrt{\frac{T}{N}} \sum_{\bar{k}, \alpha, \beta} \psi_{-, \alpha}^{*}(\bar{k}-\bar{q}) \vec{\sigma}^{\alpha \beta} \psi_{+, \alpha}(\bar{k})
$$

is the spin field at $\bar{q}=\left(q, \omega_{m}\right)$. When $\epsilon_{0}$ and $\delta U$ are absent, $g_{3}^{p}$ is real and $\theta_{ \pm}=0(\pi)$, so that $\vec{S}_{\theta_{ \pm}}$ describe $2 k_{F}$ SDW with spin maxima centered on (between) the dimers, as shown in Figure 5 . In the same Figure, for finite and positive $\epsilon_{0}$ and / or $\delta U, \theta_{ \pm}$moves away from $0(\pi)$ alongside the maxima of spin density that move in (between) the unit cell. 

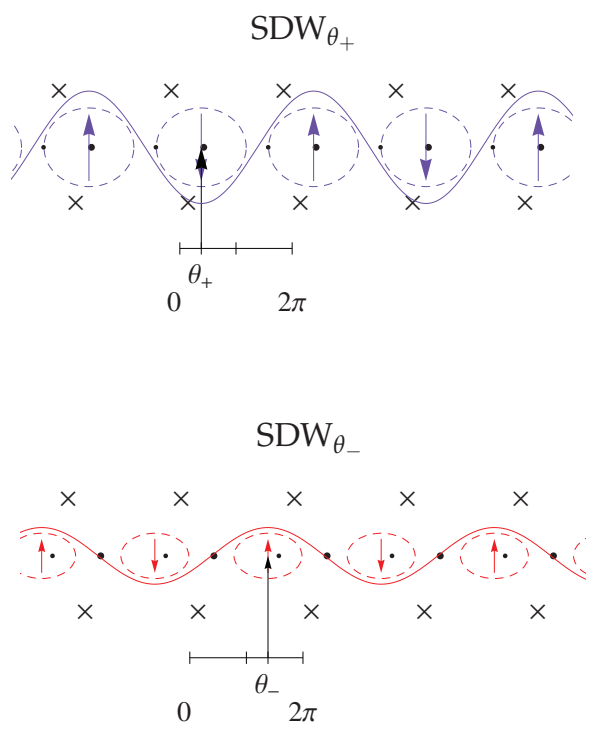

Figure 5. Site $(+)$ and bond $(-) 2 k_{F}$ spin-density-wave and their respective phase $\left(\theta_{ \pm}\right)$relative to the alternated lattice.

Making the substitution $S_{I} \rightarrow S_{I}+S_{h}$ in the RG transformation (26), the renormalized action at $\Lambda(\ell)$ reads

$$
\begin{aligned}
S\left[\psi^{*}, \psi,\{h\}\right]_{\ell} & =S_{0}\left[\psi^{*}, \psi\right]_{\ell}+S_{I}\left[\psi^{*}, \psi\right]_{\ell}+S_{h}\left[\psi^{*}, \psi,\{h\}\right]_{\ell} \\
& +\sum_{\mu, r} \chi_{\mu}^{r}\left(\bar{q}_{\mu}, \ell\right) h_{\mu}^{r *}\left(\bar{q}_{\mu}\right) h_{\mu}^{r}\left(\bar{q}_{\mu}\right)+\ldots
\end{aligned}
$$

Here, the flows of renormalization factors in $S_{0}$ and $S_{I}$ coincide with those obtained previously in (27)-(30), whereas the $z_{\mu}^{r}$ 's associated to the pair vertices in (33) are governed at the two-loop level by an equation of the form $[17,46,47]$

$$
\frac{d \ln z_{\mu}^{r}}{d \ell}=\frac{1}{2 \pi v_{F}} g_{\mu}^{r}-\frac{1}{4 \pi^{2} v_{F}^{2}}\left[g_{1}^{2}+g_{2}^{2}-g_{1} g_{2}+g_{3}^{+} g_{3}^{-} / 2\right]
$$

where for superconducting correlations $(r=\mathrm{s})$ the combinations of couplings $g_{\mu}^{r}$ are $g_{\mathrm{SS}}^{\mathrm{s}}=-g_{1}-g_{2}$ and $g_{\mathrm{TS}}^{\mathrm{s}}=g_{1}-g_{2}$ for singlet and triplet superconductivity; for density-wave correlations, one has $g_{\theta_{ \pm}}^{\mathrm{c}}=g_{2}-2 g_{1} \mp\left|g_{3}\right|$ for $\mathrm{CDW}_{\theta_{ \pm}}$in the charge sector $(r=\mathrm{c})$; and $g_{\theta_{ \pm}}^{\sigma}=g_{2} \pm\left|g_{3}\right|$ for $\mathrm{SDW}_{\theta_{ \pm}}$in the spin sector $(r=\sigma)$. The second term of (39), which is common to all pair vertices, refers to the self-energy corrections of Equation (27). According to (39), the behavior of $z_{\mu}^{r}$ is well known and follows the power law

$$
z_{\mu}^{r} \sim[\Lambda(\ell)]^{-\frac{1}{2} \gamma_{\mu}^{r *}}
$$

at large $\ell$. It signals a singularity when the exponent $\gamma_{\mu}^{r *}>0$. The expression for $\frac{1}{2} \gamma_{\mu}^{r *}$ coincides with the right side expression of (39) evaluated at the fixed points values of scaling Equations (28)-(30). A singular behavior will also be found in the corresponding expressions for susceptibilities, which are given by the quadratic field terms of (38). These are generated by the RG transformation (26) and take the form,

$$
\chi_{\mu}^{r}\left(\bar{q}_{\mu}\right)=\left(\pi v_{F}\right)^{-1} \int_{0}^{\ell}\left[z_{\mu}^{r}(\ell)\right]^{2} d \ell,
$$


which are defined positive and evaluated in the static limit $\bar{q}_{\mu}=\left(q_{\mu}, 0\right)$, for $q_{\mathrm{SS}, \mathrm{TS}}=0$ and $q_{\mathrm{CDW}, \mathrm{SDW}}=2 k_{F}$. At large $\ell$, the susceptibilities will be governed by a power law

$$
\pi v_{F} \chi_{\mu}^{r}\left(\bar{q}_{\mu}\right) \approx A_{\mu}^{r}(\ell)[\Lambda(\ell)]^{-\gamma_{\mu}^{r *}}+c_{\mu}^{r} .
$$

where $c_{\mu}^{r}$ is a positive constant.

The phase diagram determined by the dominant and subdominant singularities in the susceptibilities $\chi_{\mu}^{r}$ is shown in Figure 6, as a function of initial $g_{i}$. Its structure necessarily presents many similarities with the known two-loop RG results of the electron gas model [17,47], but also some differences due to the presence of a complex $g_{3}^{p}$. In Figure 6 the massive $\left(\Delta_{\rho} \neq 0\right)$ charge sector, delimited by the separatrix $g_{1}-2 g_{2}=\left|g_{3}\right|$, is enlarged with $\delta t, \epsilon_{0}, \delta V$ and negative $\delta U$, which is detrimental to the region of singular superconducting correlations on the left of this line. At $g_{1}>0$, this is also concomitant with the strengthening of dominant dimer or site like $\mathrm{SDW}_{\theta_{+}}$, and subdominant interdimer or bond like $\mathrm{CDW}_{\theta_{-}}$singular correlations. In the attractive region where $g_{1}<0$, only the reinforcement of $\mathrm{CDW}_{\theta_{-}}$singular correlations is found on the right-hand side of the separatrix, where a gap in both spin and charge degrees of freedom occurs.

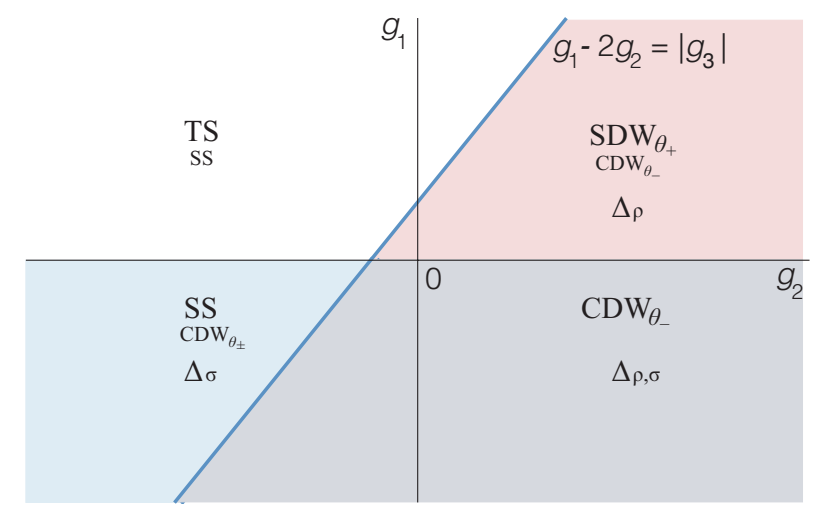

Figure 6. Phase diagram of the 1D alternated extended Hubbard model in the continuum electron gas limit.

\section{Applications}

\subsection{Anion Ordering in $\left[(T M T S F)_{1-x} \mathrm{TMTTF}_{x}\right] \mathrm{ReO}_{4}$}

As a first application of the above model, we analyze the role of CDW correlations in the mechanism of anion ordering ( $\mathrm{AO}$ ) in the concrete case of the $\mathrm{ReO}_{4}$ sulfur-selenide alloys $\left[(\mathrm{TMTSF})_{1-x} \mathrm{TMTTF}_{x}\right] \mathrm{ReO}_{4}$.

\subsubsection{Experimental Features}

In both (TMTTF) ${ }_{2} \mathrm{X}$ and (TMTSF) ${ }_{2} \mathrm{X}$ series of hole $1 / 4$-filled and dimerized compounds, with non centrosymmetric anions like $X=\mathrm{ReO}_{4}, \mathrm{NO}_{3}, \mathrm{BF}_{4} \ldots$, the possibility arises for the anion lattice to form superstructures below the ordering temperature $T_{\mathbf{q}_{\mathrm{A}}}^{0}$ [22,23]. These superstructures superimpose displacement and orientation of the anions [10]. In the high-1D-temperature domain, the most frequent staggered anion ordering wave vectors are $\mathbf{q}_{A_{1}}=\left(\frac{1}{2}, \frac{1}{2}\right)$ and $\mathbf{q}_{A_{2}}=\left(\frac{1}{2}, 0\right)$, expressed in units of the reciprocal lattice vector $\left(a^{*}, b^{*}\right)$ in the $a b$ plane of the materials (here the longitudinal part $\frac{1}{2} a^{*}=2 k_{F}$ ).

In the case of the Bechgaard salt (TMTSF) ${ }_{2} \mathrm{ReO}_{4}$, for instance, $\left(\frac{1}{2}, \frac{1}{2}\right)$ AO takes place at $T_{\frac{1}{2}, \frac{1}{2}}^{0} \simeq 177 \mathrm{~K}$ [22], which also coincides with a metal-insulator transition [48]. A similar AO superstructure is found for the Fabre salt (TMTTF) ${ }_{2} \mathrm{ReO}_{4}$ at $T_{\frac{1}{2}, \frac{1}{2}}^{0} \simeq 154 \mathrm{~K}$, a slightly lower temperature that falls within the Mott insulating state found below $T_{\rho} \simeq 230 \mathrm{~K}[30,49]$.

An interesting situation is found for the alloys [(TMTSF $\left.)_{1-x}(\text { TMTTF })_{x}\right]_{2} \mathrm{ReO}_{4}$ in solid solution [26]. At $x=0.55$, the salt is ordered with TMTSF and TMTTF molecules alternating along the stacking $a$ axis. 
Remarkably, the AO transition temperature drops and reaches the minimum, $T_{\frac{1}{2}, \frac{1}{2}}^{0} \simeq 82 \mathrm{~K}$, a value significantly lower than the pure limits at $x=0$ and $x=1$, as shown in Figure 7 [26]. By contrast, the Mott scale reaches instead a maximum at $T_{\rho}>325 \mathrm{~K}$ in the $x=0.55$ alloy [26], a value significantly higher than the two pure limits. It is worthwhile to note that the variation of $\mathrm{AO}$ temperature in the alloys differs from the one found in the hybrid salt (TMDTDSF) ${ }_{2} \mathrm{ReO}_{4}$, in which each organic molecule is composed of two sulfur and two selenium atoms [50-52]. For the latter, $T_{\frac{1}{2}, \frac{1}{2}}^{0} \simeq 165 \mathrm{~K}$, an ordering temperature which for this salt essentially falls on the midpoint between the two limits. As to the Mott scale $T_{\rho} \simeq 210 \mathrm{~K}$, it lies below the one found in the pure sulfur limit $(x=1)$.

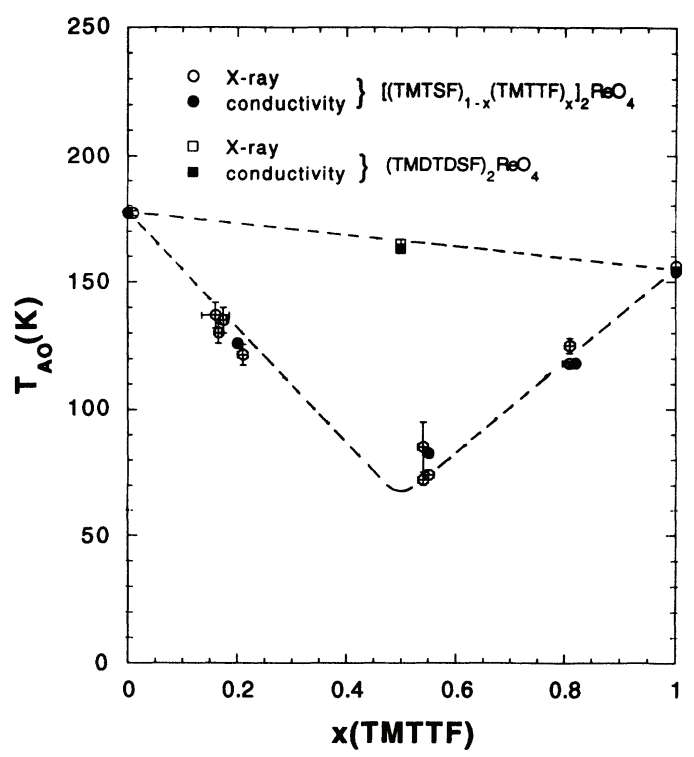

Figure 7. Anion ordering critical temperature for the $\mathrm{ReO}_{4}$ salt in different families of organic conductors and their alloys. After Ilakovac et al. [26] and references there cited.

\subsubsection{Electron-Anion Interaction}

To examine the interplay between electronic and translational anionic degrees of freedom, we add an electron-anion interaction $H_{A}$ to the purely electron part (18). Following Riera and Poilblanc [25], this interaction can be written in the form

$$
\begin{array}{r}
H_{a}=\frac{1}{2} \sum_{r, j} K_{a} \delta_{r, j}^{2}+\sum_{r, j, \sigma}\left[\left(\delta_{r, j}-\lambda \delta_{r, j-1}\right) m_{r, \sigma}\right. \\
\left.+\left(\lambda \delta_{r, j}-\delta_{r, j-1}\right) n_{r, \sigma}\right],
\end{array}
$$

where the electron charge on even and odd sites in the dimer at $r$ and chain $j$ is coupled to the anionic displacement $\delta_{r, j}$, as shown in Figure 8 . The constant $\lambda$ takes into account the two inequivalent distances between the anions and the molecular sites in the strength of the coupling strength $(0 \leq \lambda \leq 1)$. Here the anion displacements are treated in the classical harmonic approximation for which the spring constant $K_{a}$ has been rescaled $K_{a} / g^{2} \rightarrow K_{a}$ in order to incorporate the anion-electron coupling strength $g$. By using the transformations (6) and (7), the expression of $H_{a}$ for the lower band electrons, when coupled to the relevant anionic distortions $\delta_{\frac{1}{2}, q_{\perp}}$ in Fourier space, is given by

$$
\begin{aligned}
H_{a} & =\frac{1}{2} \sum_{q_{\perp}} K_{a} \delta_{\frac{1}{2}, q_{\perp}}^{2} \\
& +\frac{1}{\sqrt{N}} \sum_{p, k, q_{\perp}, \sigma} \xi_{q_{\perp}}^{p} \delta_{\frac{1}{2}, q_{\perp}} d_{p, k, \sigma}^{+} d_{-p, k-2 p k_{F}, \sigma} .
\end{aligned}
$$


The form factors for the electron-anion interaction read

$$
\xi_{0}^{p}=(\lambda-1) \frac{(t+\delta t) \epsilon_{0} / E_{F}+i p(t-\delta t)}{\sqrt{2 t^{2}+2 \delta t^{2}}},
$$

and

$$
\xi_{\frac{1}{2}}^{p}=(\lambda+1) \frac{(t+\delta t)+i p(t-\delta t) \epsilon_{0} / E_{F}}{\sqrt{2 t^{2}+2 \delta t^{2}}}
$$

for the $\left(\frac{1}{2}, 0\right)$ and $\left(\frac{1}{2}, \frac{1}{2}\right)$ anion orderings. One observes that due to the parity of the electron-anion coupling and the site potential, both factors have a real and an imaginary parts. This will then introduce a phase dependent coupling to CDW.

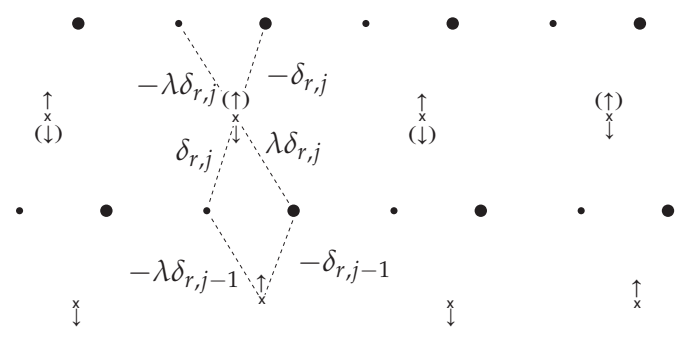

Figure 8. Electron-anion $(\times)$ interaction of the Riera-Poilblanc model [25] in systems like (TMTSF) $2 X$, $(\mathrm{TMTTF})_{2} X$ and their alloys. The arrows depict anion displacements for the $\left(\frac{1}{2}, \frac{1}{2}\right)\left[\left(\frac{1}{2}, 0\right)\right]$ ordering.

When the anionic part of the Hamiltonian is incorporated into the action $S$, it becomes

$$
S=S_{0}+S_{I}-\frac{\beta}{2} \sum_{q_{\perp}} K_{a} \delta_{\frac{1}{2}, q_{\perp}}^{2}+S_{e a}
$$

The electron-anion coupling can be recast in the form

$$
\begin{gathered}
S_{e a}=-\sqrt{\beta} \sum_{q_{\perp}} \delta_{\frac{1}{2}, q_{\perp}} e^{-i \frac{\theta_{+}}{2}}\left\{z_{\theta_{+}}^{c} O_{\theta_{+}} \operatorname{Re}\left[\xi_{q_{\perp}}^{+} e^{-i \frac{\theta_{+}}{2}}\right]\right. \\
\left.+i z_{\theta_{-}}^{c} O_{\theta_{-}} \operatorname{Re}\left[\xi_{q_{\perp}}^{+} e^{-i \frac{\theta_{-}}{2}}\right]\right\}
\end{gathered}
$$

where the renormalization factors $z_{\theta_{ \pm}}=1$ at $\ell=0$.

Therefore in the presence of a site potential $\epsilon_{0}$, which breaks the inversion symmetry within the dimer, the anion order parameter $\delta_{\frac{1}{2}, q_{\perp}}$ is coupled to the two independent $\mathrm{CDW}_{\theta_{ \pm}}$of the electron gas, as shown in Figure 4. However, there is a definite form factor for each CDW which in the end plays an important role in the type of AO stabilized. This is examined next.

\subsubsection{Anion Ordering}

The linear coupling (48) between the anion order parameter and the $\mathrm{CDW}_{\theta_{ \pm}}$composite field is similar to the one encountered in Section 3.2 for the coupling of electrons to external source fields in calculation of susceptibilities $\chi_{\mu}$. This can be exploited to generate a Landau free energy expansion of the anion order parameter. Thus considering $S_{e a}$ as a weak perturbation besides $S_{I}$, the successive partial integrations of electron degrees of freedom by the RG yield the effective action at the scale $\Lambda(\ell)$ :

$$
\begin{aligned}
S\left[\psi^{*}, \psi\right]_{\ell}=S_{0}\left[\psi^{*}, \psi\right]_{\ell}+S_{I}\left[\psi^{*}, \psi\right]_{\ell} & +S_{e a}\left[\psi^{*}, \psi\right]_{\ell} \\
& -\beta \mathfrak{F}[\delta]_{\ell} .
\end{aligned}
$$


This procedure then generates the Landau free energy $\mathfrak{F}[\delta]_{\ell}$ of the anionic order parameter. Up to the quadratic level it takes the form

$$
\mathfrak{F}[\delta]_{\ell}=\sum_{q_{\perp}}\left[K_{a} / 2-\chi_{\frac{1}{2}, q_{\perp}}(\ell)\right] \delta_{\frac{1}{2}, q_{\perp}}^{2}+\mathcal{O}\left(\delta^{4}\right),
$$

where

$$
\chi_{\frac{1}{2}, q_{\perp}}(\ell)=\sum_{\mu= \pm} \operatorname{Re}\left[\xi_{q_{\perp}}^{+} e^{-i \frac{\theta_{\mu}}{2}}\right] \chi_{\theta_{\mu}}^{c}(\ell)
$$

is the effective susceptibility involved in the $\mathrm{AO}$ at $\left(\frac{1}{2}, q_{\perp}\right)$. Relating the loop variable $\ell=\ln E_{F} / T$ to temperature, the zero of the quadratic coefficient leads to the following condition for the AO critical temperature $T_{\frac{1}{2}, q_{\perp}}^{0}$,

$$
\frac{K_{a}}{2}=\chi_{\frac{1}{2}, q_{\perp}}\left(T_{\frac{1}{2}, q_{\perp}}^{0}\right)
$$

The strongest anion susceptibility will lead to the highest $T_{\frac{1}{2}, q_{\perp}}^{0}$. For repulsive interactions $U=2 V>0$ and non zero $K_{a}$, the regions of stability of both $\left(\frac{1}{2}, \frac{1}{2}\right)$ and $\left(\frac{1}{2}, 0\right) \mathrm{AO}$ in the $\left(\delta t, \epsilon_{0}\right)$ plane are shown in Figure 9 for different values of $\lambda$.

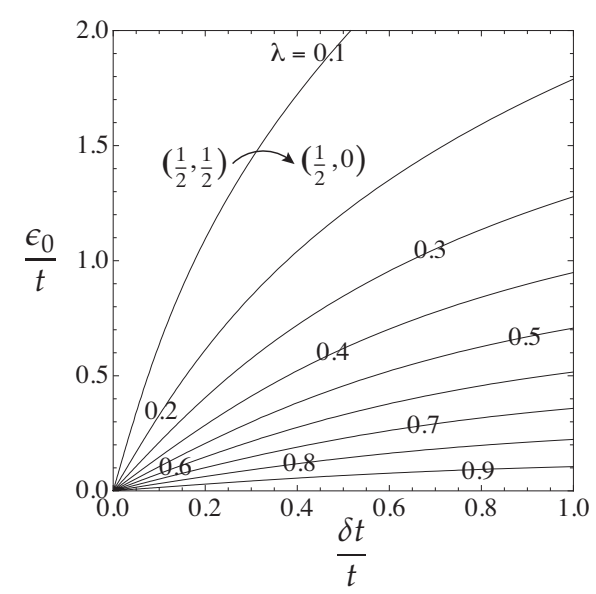

Figure 9. Iso $-\lambda$ phase boundaries between $\left(\frac{1}{2}, \frac{1}{2}\right)$ and $\left(\frac{1}{2}, 0\right)$ anion orderings, as a function of the normalized site potential and dimerization.

It is useful to first consider limiting cases for the asymmetry parameter $\lambda$ related to the position of the anions in the unit cell. Thus for $\lambda \rightarrow 1$, each anion is aligned with the center of a dimer and $\xi_{0}^{+} \rightarrow 0$, so that only the $\left(\frac{1}{2}, \frac{1}{2}\right)$ ordering is stabilized through a coupling to both $\mathrm{CDW}_{\theta_{ \pm}}$. Interestingly, the most important coupling is to the $\mathrm{CDW}_{\theta_{+}}$correlations which according to Figure 6 are not singular, at variance with $\mathrm{CDW}_{\theta_{-}}$. This indicates that the driving force of the $\left(\frac{1}{2}, \frac{1}{2}\right) \mathrm{AO}$ is not the result of a $2 k_{F}$ instability of the electron gas, as it is for the ordinary Peierls mechanism.

In the opposite decentered limit, where $\lambda \rightarrow 0$, both anion couplings are finite. At $\delta t=0$, they becomes equal in amplitude and couple identically to both $\mathrm{CDW}_{\theta_{ \pm}}$. The phase boundary then merges with the $\epsilon_{0}$ axis at $\delta t=0$, where $\theta_{+}=\pi / 2$ and $\theta_{-}=-\pi / 2$ (or $\left.3 \pi / 2\right)$ and anions are coupled to completely site centered CDW (see Figure 4). It follows that for such a $\lambda$, the $\left(\frac{1}{2}, 0\right)$ phase is stable over the whole $\left(\delta t>0, \epsilon_{0}\right)$ plane. In this case, the singular $\mathrm{CDW}_{\theta_{-}}$correlations are the main driving force of $\mathrm{AO}$.

When $\lambda$ grows from zero in Figure 9, the region of stability of $\left(\frac{1}{2}, \frac{1}{2}\right)$ phase starts to increase against that of $\left(\frac{1}{2}, 0\right)$. According to (45) and (46), this is the consequence of a reinforcement (weakening) of the effective anion coupling $\operatorname{Re}\left[\xi_{\frac{1}{2}}^{+} e^{-i \theta_{ \pm} / 2}\right]\left(\operatorname{Re}\left[\xi_{0}^{+} e^{-i \theta_{ \pm} / 2}\right]\right)$ to the $\mathrm{CDW}_{\theta_{ \pm}}$. For the whole range of $\lambda$, 
the $\left(\frac{1}{2}, \frac{1}{2}\right) \mathrm{AO}$ is essentially due to the coupling to the non singular $\mathrm{CDW}_{\theta_{+}}$, whereas the $\left(\frac{1}{2}, 0\right) \mathrm{AO}$ is mainly coupled to the singular $\mathrm{CDW}_{\theta_{-}}$.

\subsubsection{Theory and Experiment}

One can proceed to the application of the above model to the variation of anion ordering $T_{\frac{1}{2}, \frac{1}{2}}$ and Mott insulating scale $T_{\rho}$ in the alloys [(TMTTF) $)_{1-x} \mathrm{TMTSF}_{x}$ ] $\mathrm{ReO}_{4}$. First, we fix the various parameters of the model. The average longitudinal hopping $t$ of the model can be set from the results of band calculations $[53,54], t=[364(1-x)+200 x] \mathrm{meV}$, interpolating between the pure Fabre $(x=1)$ and the Bechgaard $(x=0)$ limits. From these results, one can also determine the modulation of the hopping, $\delta t=[26(1-x)+14.5 x] \mathrm{meV}$. From photoemission experiments [55], the difference in ionization energy between the TMTSF and TMTTF organic molecules leads to a site modulation energy of $\epsilon_{0} \simeq 200 \mathrm{meV}$, in order to use $\epsilon_{0}(x)=4 x(1-x) 200 \mathrm{meV}$, as the effective alternating site potential as a function of $x$ (this amplitude for the site potential $\epsilon_{0}$ does not include the contribution coming from charge ordering, which is observed in (TMTTF) ${ }_{2} \mathrm{ReO}_{4}$ at $x=1[30,49]$ and should be present at finite $x$. Although this contribution is not known in the alloy, its input in the calculations would further suppress anion ordering transition in Figure 10). Regarding interactions, we shall take $U=[200(1-x)+268 x] \mathrm{meV}$ giving a stronger (weaker) site repulsive in the Fabre (Bechgaard) case. According to quantum chemistry calculations [44], the value of the nearest-neighbor interaction can be fixed at $V=U / 2$ for all $x$. Finally, we shall link the small modulations of interactions, $\delta U=-\frac{\epsilon_{0}}{E_{F}} \frac{U}{3}$ and $\delta V=\frac{t \delta t}{E_{F}^{2}} \frac{U}{3}$, to those of $\epsilon_{0}, U$ and $\delta t$. In this way for instance, the values $U \pm \delta U$ and $V \pm \delta V$ at $x=0.5$ corresponds to the interaction values in the Bechgaard $(+)$ and Fabre $(-)$ cases.

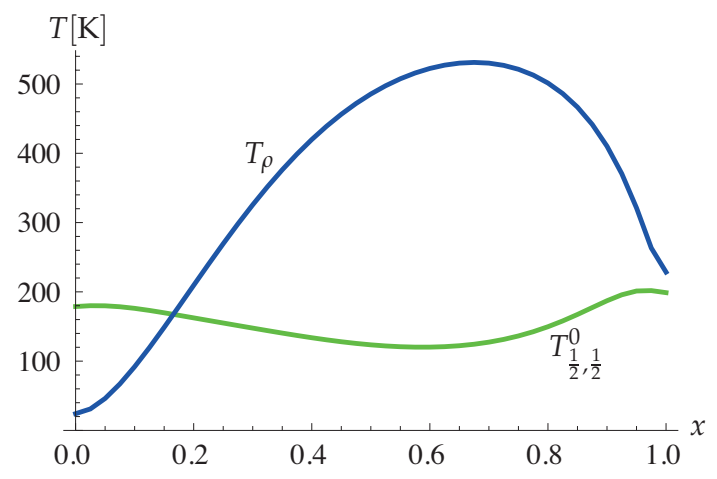

Figure 10. Calculated critical temperature for the $\left(\frac{1}{2}, \frac{1}{2}\right)$ anion ordering (green) and Mott scale (blue), as a function of TMTTF concentration $x$ in $\left[(\mathrm{TMTSF})_{1-x}(\mathrm{TMTTF})_{x}\right]_{2} \mathrm{ReO}_{4}$ alloys.

With the above figures, the $\left(\frac{1}{2}, \frac{1}{2}\right)$ AO will dominate for not too small $\lambda$ and essentially arbitrary values of anion lattice stiffness $K_{a}$. We shall fix $K_{a} /\left[2(1+\lambda)^{2}\right]=1.75(\mathrm{eV})^{-1}$ to give from (52), the experimental value $T_{\frac{1}{2}, \frac{1}{2}}^{0} \simeq 180 \mathrm{~K}$ in the Bechgaard $x=0$ limit [22]. Thus when the site potential $\epsilon_{0}(x)$ grows with $x$, alongside the effect of dimerization $\delta t / t, T_{\frac{1}{2}, \frac{1}{2}}^{0}$ decreases due to the weakening of the coupling to non singular $\mathrm{CDW}_{\theta_{+}}$correlations in (51) and (52); it reaches a minimum at $x=0.5$, namely where $\epsilon_{0}$ is maximum, in qualitative agreement with the results of Figure 7. As $x$ grows further, $\epsilon_{0}$ goes down and $T_{\frac{1}{2}, \frac{1}{2}}^{0}$ starts to increase and evolve toward the limiting value of $190 \mathrm{~K}$ in the $x=1$ Fabre case. This value is nearly the same than for $x=0$, but higher than the experimental value $[30,49,56]$. The minimum value $T_{\frac{1}{2}, \frac{1}{2}}^{0} \simeq 105 \mathrm{~K}$ at $x=0.5$ corresponds to a decrease of $40 \%$ or so from the pure $x=0$ value, compared with nearly $55 \%$ for experiments in Figure 7 , showing a qualitative agreement (see the above note in Section 4.1.4).

In contrast to $T_{\frac{1}{2}, \frac{1}{2}}^{0}$, the value of the Mott scale $T_{\rho}$ in Figure 10 reaches a maximum at more than twice its value found in the $x=1$ Fabre limit. This is a consequence of positive $\epsilon_{0}$ and $\delta t / t$ 
alternating potentials, whose influences add in quadrature in the $g_{3}$ expression in (19) and the value of $T_{\rho}$. This conclusion for $T_{\rho}$ agrees with those previous works [26-29].

\subsection{Interplay between the Spin-Peierls and Charge Ordered States}

As a second application of our model, we examine the influence of charge ordering on the spin-Peierls instability of weakly localized 1D Mott insulators of the Fabre salts series.

\subsubsection{Experiments}

The spin-Peierls instability is found in some members of the Fabre (TMTTF) ${ }_{2} X$ series with $\mathrm{X}=\mathrm{PF}_{6}$ and $\mathrm{AsF}_{6}$. These quarter-filled band but weakly dimerized systems show a 1D Mott insulating behavior below some temperature scale $T_{\rho} \simeq 220 \mathrm{~K}[57,58]$. In (TMTTF) ${ }_{2} \mathrm{AsF}_{6}$, for instance, the Mott insulating behavior is followed at lower temperature by a continuous transition toward the formation of charge ordered $(\mathrm{CO})$ state at $T_{\mathrm{CO}} \simeq 103 \mathrm{~K}[29,31]$. Below $T_{\mathrm{CO}}$, there is a charge disproportionation in the dimer unit cell leading to a finite static alternated site potential $\epsilon_{0}$. Within the CO state, there is an additional instability that involves both spins and lattice degrees of freedom. According to x-ray diffuse scattering [37,39], the lattice becomes unstable with the onset of 1D lattice fluctuations at the wave vector $2 k_{F}$ below the characteristic temperature scale $T_{\mathrm{SP}}^{0}$. For the $\mathrm{AsF}_{6} \mathrm{compound}$ $T_{\mathrm{SP}}^{0} \simeq 40 \mathrm{~K}[10,37]$. Occurring well below the Mott scale $T_{\rho}, T_{\mathrm{SP}}^{0}$ takes place in the presence of strong antiferromagnetic correlations and then refers to a spin-Peierls (SP) instability. A true 3D SP ordering, however, occurs only at a much lower temperature, namely, $T_{\mathrm{SP}} \simeq 11 \mathrm{~K}[37,39]$. The latter obeys the empirical rule $T_{\mathrm{SP}} \sim T_{\mathrm{SP}}^{0} / 3$ for the reduction of the ordering temperature by $1 \mathrm{D}$ fluctuations in weakly coupled Peierls and spin-Peierls chains.

From NMR experiments under pressure [39], $T_{\mathrm{SP}}$ is found to increase while $T_{\mathrm{CO}}$ is steadily decreasing. This indicates that a reduction of the charge disproportionation, that is the site alternated potential $\epsilon_{0}$, enhances the SP ordering. The increase of $T_{\mathrm{SP}}$ with pressure carries on until its amplitude reaches $T_{\mathrm{CO}}$, defining a critical pressure above which $T_{\mathrm{SP}}$ undergoes a steady decrease (see Figure 11).

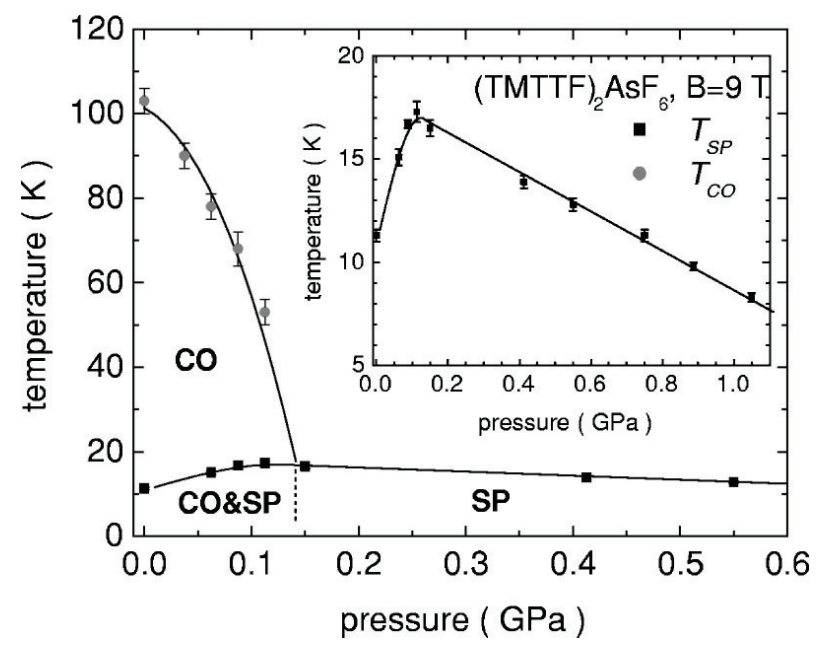

Figure 11. Critical temperatures of spin-Peierls and charge ordered states as a function of applied pressure in (TMTTF) ${ }_{2} \mathrm{AsF}_{6}$, as determined from NMR experiments. From Zamborszky et al. [39].

\subsubsection{Electron-Lattice Coupling}

The formation of a bond superstructure in the dimerized chains results from a coupling of the lattice degrees of freedom to bond charge density-wave correlations of electrons. By analogy with a Peierls electron-lattice coupling in a tight-binding band $[59,60]$, the coupling develops from the modulation of the interdimer hopping integral $t_{2}=t-\delta t$ by the displacement $\phi_{r}$ of the dimer from its 
equilibrium position. The expansion of $t_{2}(\phi)$ with respect to $\phi$ leads to an additional electron-lattice part to the Hamiltonian (5) which is of the form,

$$
\begin{aligned}
H_{\mathrm{ep}}+H_{\mathrm{p}}^{0}= & \sum_{r, \sigma} t_{2}^{\prime}\left[a_{r, \sigma}^{\dagger} b_{r-1, \sigma}\left(\phi_{r-1}-\phi_{r}\right)+\text { H.c. }\right] \\
& +\frac{K_{D}}{2} \sum_{r}\left(\phi_{r-1}-\phi_{r}\right)^{2} .
\end{aligned}
$$

Here $t_{2}^{\prime}=d t_{2} / d \phi$ and $K_{D}$ is the spring constant of the harmonic potential energy, $H_{\mathrm{p}}^{0}$, of interdimer lattice modes in the static limit. From the canonical transformation (6) and (7), the Hamiltonian for the coupling of the tetramerization order parameter, $\phi_{2 k_{F}}$, to electrons close to Fermi level is given by

$$
\begin{aligned}
H_{\mathrm{ep}}+H_{\mathrm{p}}^{0}= & \frac{1}{\sqrt{N}} \sum_{k, \sigma}\left[g_{\mathrm{ep}}\left(\epsilon_{0}\right) d_{+, k, \sigma}^{\dagger} d_{-, k-2 k_{F}, \sigma} \phi_{2 k_{F}}+\text { H.c. }\right] \\
& +2 K_{D}\left|\phi_{2 k_{F}}\right|^{2}
\end{aligned}
$$

where

$$
g_{\mathrm{ep}}\left(\epsilon_{0}\right)=-2 i t_{2}^{\prime} \sin \gamma_{k_{F}}=-2 i t_{2}^{\prime} \sqrt{1-\frac{\epsilon_{0}^{2}}{E_{F}^{2}}}
$$

is the electron-lattice coupling constant showing an explicit dependence on the potential amplitude $\epsilon_{0}$ of $\mathrm{CO}$, which reduces the strength of the coupling.

Transposing this term into the action allows to write at $\ell=0$,

$$
S=S_{0}+S_{I}-2 \beta K_{D}\left|\phi_{2 k_{F}}\right|^{2}+S_{\mathrm{ep}}
$$

where

$$
\begin{aligned}
S_{\mathrm{ep}}=-\sqrt{\beta} g_{\mathrm{ep}}\left(\epsilon_{0}\right)\left[\left(z_{\theta_{+}}^{c} O_{\theta_{+}} \sin \frac{1}{2} \theta_{+}\right.\right. \\
\left.\left.-i z_{\theta_{-}}^{c} O_{\theta_{-}} \sin \frac{1}{2} \theta_{-}\right) \phi_{2 k_{F}}+\text { c.c. }\right],
\end{aligned}
$$

in which we have decomposed the BOW composite field in terms of the two independent CDW at $\theta_{ \pm}=\theta_{g_{3}}\left(\theta_{g_{3}} \pm \pi\right)$, and for $q_{0}=\left(2 k_{F}, 0\right)$. Here the initial conditions for the pair vertex renormalization factors at $\ell=0$ are $z_{\theta_{ \pm}}^{c}=1$.

\subsubsection{Spin-Peierls Instability}

The presence of the SP order parameter $\phi_{2 k_{F}}$ then linearly couples as an external field to the two independent CDW for a complex $g_{3}$. In weak coupling $S_{\text {ep }}$ can be taken as a perturbative term alongside $S_{I}$. Thus the successive partial integrations of the RG transformation following (26), down to the energy distance $\Lambda_{0}(\ell)$ from the Fermi level will lead to the effective action at step $\ell$ :

$$
\begin{gathered}
S\left[\psi^{*}, \psi\right]_{\ell}=S_{0}\left[\psi^{*}, \psi\right]_{\ell}+S_{I}\left[\psi^{*}, \psi\right]_{\ell}+S_{\mathrm{ep}}\left[\psi^{*}, \psi, \phi\right]_{\ell} \\
-\beta \mathfrak{F}[\phi]_{\ell} .
\end{gathered}
$$

The RG then generates a $\ell$ dependent Landau free energy $\mathfrak{F}[\phi]_{\ell}$ of the SP order parameter. At the quadratic level, it takes the form

$$
\begin{gathered}
\mathfrak{F}[\phi]_{\ell}=\left[2 K_{D}-\left|g_{\mathrm{ep}}\left(\epsilon_{0}\right)\right|^{2} \sum_{\mu= \pm}\left(\sin ^{2} \frac{1}{2} \theta_{\mu}\right) \chi_{\theta_{\mu}}^{c}\left(q_{0}, \ell\right)\right]\left|\phi_{2 k_{F}}\right|^{2} \\
+\mathcal{O}\left(\phi^{4}\right) .
\end{gathered}
$$


The mean field criteria for the SP 'transition' temperature at $T_{\mathrm{SP}}^{0}$ is obtained from the zero of the quadratic term at $\ell_{\mathrm{SP}}^{0}=\ln E_{F} / T_{\mathrm{SP}}^{0}$. This leads to

$$
\frac{2 K_{D}}{\left|g_{\mathrm{ep}}\left(\epsilon_{0}\right)\right|^{2}}=\sum_{\mu= \pm}\left(\sin ^{2} \frac{1}{2} \theta_{\mu}\right) \chi_{\theta_{\mu}}^{c}\left(q_{0}, T_{\mathrm{SP}}^{0}\right)
$$

The right-hand-side of this expression is essentially dominated by the $\mathrm{CDW}_{\theta_{-}}$susceptibility $\chi_{\theta_{-}}^{c}$ which is more centered on bonds. It presents a power law divergence with decreasing temperature, while $\chi_{\theta_{+}}^{c}$, which is more site-centered saturates at a small value. According to the results of Section 3.2, the bond susceptibility takes the form

$$
\chi_{\theta_{-}}^{c}\left(q_{0}, T\right) \sim\left(\pi v_{F}\right)^{-1} C_{\theta_{-}}\left(T / T_{\rho}\right)^{-\gamma^{*}},
$$

where $\gamma^{*}=3 / 2$ for the fixed point behavior below the Mott scale $T_{\rho}$ [47]. Here $C_{\theta_{-}}$is a positive constant that gives the contribution to the susceptibility from all energy scales above $T_{\rho}$.

By singling out the dominant $\theta_{-}$part, we obtain the approximate result for the mean field SP temperature

$$
T_{\mathrm{SP}}^{0} \approx\left[C_{\theta_{-}}\left(\sin ^{2} \frac{1}{2} \theta_{-}\right)\left|\bar{g}_{\mathrm{ep}}\left(\epsilon_{0}\right)\right|^{2} / 2 K_{D}\right]^{\frac{2}{3}} T_{\rho},
$$

where $\left|\bar{g}_{\mathrm{ep}}\left(\epsilon_{0}\right)\right|^{2}=\left|g_{\mathrm{ep}}\left(\epsilon_{0}\right)\right|^{2} / \pi v_{F}$. Although from (21) an increase of the site potential $\epsilon_{0}$ raises the value of $\left|g_{3}\right|$ and then $T_{\rho}, \epsilon_{0}$ is a major source of reduction of the electron-phonon matrix element $\bar{g}_{\mathrm{ep}}\left(\epsilon_{0}\right)$, which together with the shift of $\mathrm{CDW}_{\theta_{-}}$off the bonds, leads to an overall decrease of $T_{\mathrm{SP}}^{0}$ with $\epsilon_{0}$. This is shown in Figure 12 (top). The charge imbalance between sites of neighbouring dimers is therefore acting as the main source of reduction of the lattice coupling to $2 k_{F}$ bond correlations.
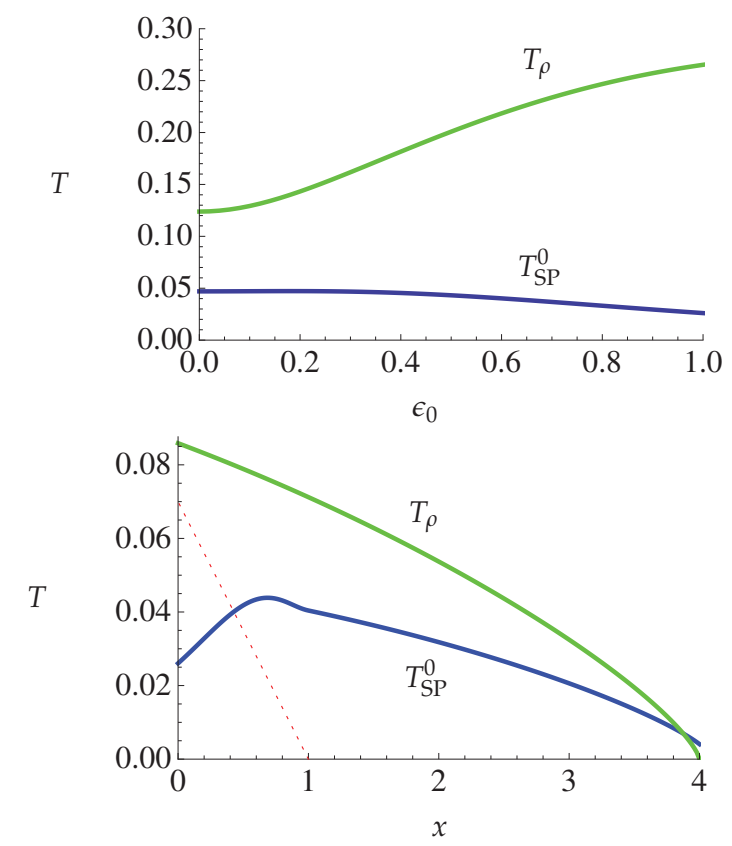

Figure 12. (top): Calculated variation of the mean-field SP ordering temperature $T_{\mathrm{SP}}^{0}$ and of the Mott scale $T_{\rho}$ with the amplitude of site potential $\epsilon_{0}$ due to charge ordering; (bottom): Calculated variations of the mean-field $1 \mathrm{D}(3 \mathrm{D})$ spin-Peierls temperature $T_{\mathrm{SP}}^{0}\left(T_{\mathrm{SP}} \sim T_{\mathrm{SP}}^{0} / 3\right)$, as a function of the tuning (pressure) parameter $x$. The dashed red line is a linear parametrization of the charge ordering temperature $T_{\mathrm{CO}}=\epsilon_{0}(x) / 2$. All temperature scales are normalized by the average hopping $t$ along the stacks. 
It is worth noticing that the mean-field prediction $T_{\mathrm{SP}}^{0}$ does not coincide with a real transition temperature, but refers to the temperature scale for the onset of strong $2 k_{F}$ 1D lattice SP fluctuations [61,62], such as those seen for instance in diffuse x-ray scattering [37]. For a true 3D transition to take place, interchain coupling $V_{\perp}$ (e.g., electron-lattice, Coulomb, ...) is necessary to stabilize long-range ordering for a one-component SP order parameter. The generalization of the above approach to the case of weakly coupled Landau-Ginzburg chains is well known [63]; it leads to the relation

$$
T_{\mathrm{SP}} \simeq \frac{T_{\mathrm{SP}}^{0}}{\ln \left(T_{\mathrm{SP}}^{0} / V_{\perp}\right)} \sim T_{\mathrm{SP}}^{0} / \alpha,
$$

between the $T_{\mathrm{SP}}^{0}$ and $T_{\mathrm{SP}}$ scales. For $V_{\perp} \ll T_{\mathrm{SP}}^{0}$, fluctuations reduce $T_{\mathrm{SP}}^{0}$ by a factor $\alpha$, which is typically around 3 .

\subsubsection{Theory and Experiment}

We are now in a position to apply our model to the evolution of the SP instability in the presence of $\mathrm{CO}$ for a compound like (TMTTF) ${ }_{2} \mathrm{AsF}_{6}$ under pressure (top of Figure 12). We consider the hopping modulation $\delta t(x)=0.2-0.5 x$ (normalized by $t=1$ ), tuned downward by the pressure parameter $x$. Here, 0.2 is a typical band calculation value of $\delta t$ for a compound like (TMTTF) $)_{2} \mathrm{AsF}_{6}$ at ambient pressure $(x=0)$ [64]. We fix the interaction parameters $U=1$ and $V=0.5$ in order to obtain a Mott temperature scale, $T_{\rho} \sim 100 \mathrm{~K}\left(T_{\rho} \sim 0.09\right.$ in units of $\left.t\right)$, consistent with the range found in experiments for a value of $t=1300 \mathrm{~K}$ at ambient pressure [64]. Charge ordering is responsible for the onset of a static site potential, which we parametrize by the linear profile $\epsilon_{0}(x)=\epsilon_{0}(0)\left(1-x / x_{c}\right)$. Here we shall use the relation $\epsilon_{0}(0)=\Delta_{\mathrm{CO}} \sim 2 T_{\mathrm{CO}}$ between the $\mathrm{CO}$ gap and the observed critical temperature $T_{\mathrm{CO}} \approx 100 \mathrm{~K}$ at ambient pressure. Finally, we let the normalized small variations $\delta U=-\epsilon_{0}(x) / E_{F}$ and $\delta V=t \delta t(x) / E_{F}^{2}$ evolving under pressure following $\epsilon_{0}(x)$ and $\delta t(x)$, respectively.

The solution of (60) for $T_{\mathrm{SP}}^{0}$ from the use of (42) and (55) as a function of the tuning parameter $x$, is displayed in the bottom panel of Figure 12. We see that to the fall of $\epsilon_{0}(x)$ under pressure corresponds an increase of $T_{\mathrm{SP}}^{0}$, the latter being a consequence of the boost in the electron lattice-coupling $g_{\mathrm{ph}}\left(\epsilon_{0}\right)$ that overcomes the impact of the reduction of $T_{\rho}$ on $T_{\mathrm{SP}}^{0}$ under pressure. A maximum of $T_{\mathrm{SP}}^{0}$ is reached at $x_{c}$ where $\epsilon_{0}\left(x_{c}\right)$ vanishes, leading to near $50 \%$ of increase of $T_{\mathrm{SP}}^{0}$ from its ambient pressure value. Beyond $x_{c}, \epsilon_{0}\left(x>x_{c}\right)=0$ and $T_{S P}^{0}$ undergoes a monotonic decreases with $x$, which according to (60) and (61) is governed by that of $T_{\rho}$ under pressure-when only bond order wave correlations with $\theta_{-}=\pi$ couple to the lattice (at sufficiently high but intermediate pressure, interchain antiferromagnetic exchange coupling, which is present but not considered in the present high temperature calculations, enters into play and introduces a competition between the SP and the magnetic Néel states, from which the latter state ends up to be favored $[20,21,61]$. This competition is also documented on experimental grounds in the similar compound (TMTTF) ${ }_{2} \mathrm{PF}_{6}$ - see for instance Refs. $[65,66]$. In the very high pressure range, it is the turn of the Mott scale $T_{\rho}$ to become irrelevant in (TMTTF) ${ }_{2} X$, with the Néel state merging into an itinerant SDW state when interchain exchange evolves towards coherent interchain hopping, as found in (TMTSF) ${ }_{2} X$ at low pressure [1,4]. In these conditions the absence of a tetramerization-Peierls-instability, despite the absence of charge ordering, may take its origin from the relatively weak amplitude of Umklapp scattering. This suppresses $T_{\rho}$ and strongly reduces the growth of bond CDW correlations for the Peierls scale $T_{P}^{0}$ in (60). The long-range Peierls ordering is likely to be preempted when conflicting sources of interchain coupling are added to the model such as single electron hopping and Coulomb interactions, along with the emergence of SDW long-range order [10]).

The results of the above model for $T_{\mathrm{SP}}^{0}$ and by extension $T_{\mathrm{SP}}$ fairly bear the comparison with the experiments shown in Figure 11. The present model could also offer an explanation as to the absence of a spin-Peierls transition in a compound like (TMTTF) ${ }_{2} \mathrm{SbF}_{6}$, which rather exhibits a Néel transition around $7 \mathrm{~K}$ [67]. The $\mathrm{SbF}_{6} \mathrm{Fabre}$ salt is known to develop charge ordering at a temperature $T_{\mathrm{CO}} \approx 160 \mathrm{~K}[29,30,68]$, which is sizably higher than for other SP salts like $\mathrm{PF}_{6}$ and 
$\mathrm{AsF}_{6}$. Following (55), a significantly larger $T_{\mathrm{CO}}$ or $\epsilon_{0}$, together with a relatively weaker value of $T_{\rho}$ for $\mathrm{SbF}_{6}$ [58], would further suppress the electron-phonon matrix element $g_{\mathrm{ep}}\left(\epsilon_{0}\right)$ and from (61) the scales $T_{\mathrm{SP}}^{0}$ and $T_{\mathrm{SP}}$. Including the influence of interchain exchange interaction between spins, which is present in practice but has been disregarded in this work (see for instance Refs. [20,21,61]), will favor the competition between the SP and the Néel states and may be responsible for the absence of long-range SP ordering and the relatively small Néel temperature for this salt (see also the note in the preceding paragraph). The observation of a reentrant $\mathrm{SP}$ state in (TMTTF $)_{2} \mathrm{SbF}_{6}$ at higher pressure is consistent with that view [67]. These results obtained above are also compatible with those using the bosonization technique [40], and numerics $[35,40]$.

\section{Concluding Remarks}

In the work developed above, we inquired into the properties of the one-dimensional extended Hubbard model at quarter-filling with superimposed dimerization, site and anion alternating lattice potentials, as they can be found in practice in low-dimensional charge transfer salts with a 2:1 stochiometry. The renormalization group method was applied to the continuum or electron gas limit of the model in order to determine the influence of dimerization and site commensurability potentials on low energy interactions, in particular effective half-filling type of Umklapp scattering that emerges from the lattice. The impacts of Umklapp on singular correlations and different excitation gaps of the model have been determined, along with spin and charge density-wave correlations and their specific phase relations to the underlying alternated lattice.

By coupling electrons to anionic displacements in systems like the (TMTTF) $)_{2} X$ and (TMTSF) $2 X$ charge transfer salts, the mechanism of anion ordering in these materials has been investigated. It was found that a site alternated potential can significantly reduce the coupling of charge density wave correlations to anion displacements and in turn the critical temperature of staggered anion ordering. This occurs while the Mott insulating character of electrons is on the contrary strongly enhanced by site and bond commensurabilities. These opposed effects were found to be consistent with observations about anionic order and Mott insulating state in $\left[(\mathrm{TMTSF})_{1-x}(\mathrm{TMTTF})_{x}\right]_{2} \mathrm{ReO}_{4}$ alloys.

The influence of the charge ordered state on the spin-Peierls ordering has also been investigated for members of the Fabre salts series at low applied pressure. Acting as alternate site commensurability, charge order was found to mainly reduce the inter-dimer tight-binding electron-phonon matrix element. The coupling of the lattice to bond density-wave correlations is then lessened by charge disproportionation and a competition between the spin-Peierls and the charge ordered states takes place. The interplay gives rise to a characteristic dome of the spin-Peierls ordering temperature as a function of the suppression of charge order under pressure, a result congruent with observations made in the Fabre salts series.

Allowing for anionic displacements besides lattice degrees of freedom would be an interesting possible extension of the latter calculations. This could provide the opportunity to check if the spin-Peierls lattice distortion is accompanied by staggered anion ordering, as suspected on experimental grounds for spin-Peierls systems of the Fabre salts series [11].

It is worth mentioning another straightforward application of the model of anion ordering which was not considered above. This concerns the anion displacement that goes with the charge ordering transition in the (TMTTF) ${ }_{2} X$ series. Such anion displacement is known to be uniform in character [10]. The corresponding order parameter $\delta_{0,0}$ will thus be linearly coupled to uniform charge density of the stacks, in a way similar to the expression given in (48). Since uniform charge susceptibility is a quantity proportional to the dielectric constant, as the latter is being singular at the charge ordering transition $[32,68]$, it will drive a collective shift of the anionic position as apparently found experimentally $[9,68,69]$.

Author Contributions: M.M. performed the calculations and the comparisons with experiments in the framework of a PhD thesis at Universite de Sherbrooke (2017). C.B. wrote the paper. Both authors contributed to the discussion of the results. All authors have read and agreed to the published version of the manuscript. 
Funding: This research was funded by the National Science and Engineering Research Council of Canada (NSERC) and the Réseau Québcois des Matériaux de Pointe (RQMP).

Acknowledgments: The authors thank G. Chitov for useful comments on many aspects of this work.

Conflicts of Interest: The authors declare no conflict of interest.

\section{References}

1. Jérome, D.; Schulz, H.J. Organic conductors and superconductors. Adv. Phys. 1982, 31, 299. [CrossRef]

2. Ishiguro, T.; Yamaji, K. Organic Superconductors; Springer-Verlag Series in Solid-State Science; Springer: Berlin/Heidelberg, Germeny, 1990; Volume 88.

3. Bourbonnais, C.; Jérome, D. The normal phase of quasi-one-dimensional organic superconductors. In Advances in Synthetic Metals, Twenty Years of Progress in Science and Technology; Bernier, P., Lefrant, S., Bidan, G., Eds.; Elsevier: New York, NY, USA, 1999; pp. 206-261.

4. Bourbonnais, C.; Jérome, D. Interacting electrons In quasi-one-Dimensional organic superconductors. In The Physics of Organic Superconductors and Conductors; Springer Series in Materials Science; Lebed, A., Ed.; Springer: Heidelberg, Germany, 2008; Volume 110, p. 357.

5. Brown, S.E.; Chaikin, P.M.; Naughton, M.J. La tour des sels de Bechgaard. In The Physics of Organic Superconductors and Conductors; Springer Series in Materials Science; Lebed, A., Ed.; Springer: Heidelberg, Germany, 2008; Volume 110, p. 49.

6. Seo, H.; Hotta, C.; Fukuyama, H. Towards systematic understanding of diversity of electronic properties in low-dimensional molecular solids. Chem. Rev. 2004, 104, 5005. [CrossRef] [PubMed]

7. Brown, S.E. The Bechgaard salts and relatives. Physica C 2015, 514, 279. [CrossRef]

8. Dressel, M. Ordering phenomena in quasi-one-dimensional organic conductors. Naturwissenschaften 2007, 94, 527. [CrossRef] [PubMed]

9. Dressel, M.; Dumm, M.; Knoblauch, T.; Masino, M. Comprehensive optical investigations of charge order in organic chain compounds (TMTTF) ${ }_{2} X$. Crystals 2012, 2, 528. [CrossRef]

10. Pouget, J.P. Interplay between electronic and structural degrees of freedom in quarter-filled low dimensional organic compounds. Physica B 2015, 460, 45. [CrossRef]

11. Pouget, J.P.; Alemany, P.; Canadell, E. Donor-anion interactions in quarter-filled low-dimensional organic conductors. Mater. Horizons 2018, 5, 590. [CrossRef]

12. Rosslhuber, R.; Rose, E.; Ivek, T.; Pustogow, A.; Breier, T.; Geiger, M.; Schrem, K.; Untereiner, G.; Dressel, M. Structural and electronic properties of (TMTTF $)_{2} \mathrm{X}$ salts with tetrahedral anions. Crystals 2018, 8, 121. [CrossRef]

13. Clay, R.T.; Mazumdar, S. From charge- and spin-ordering to superconductivity in the organic charge-transfer solids. Phys. Rep. 2019, 788, 1. [CrossRef]

14. Barisic, S.; Brazovskii, S. Recent Developments in Condensed Matter Physics; Devreese, J.T., Ed.; Plenum: New York, NY, USA, 1981; Volume 1, p. 327.

15. Emery, V.J.; Bruinsma, R.; Barisic, S. Electron-electron umklapp scattering in organic superconductors. Phys. Rev. Lett. 1982, 48, 1039. [CrossRef]

16. Dzyaloshinskii, I.E.; Larkin, A.I. Possible states of quasi-unidimensional systems. Sov. Phys. JETP 1972, 34, 422.

17. Solyom, J. The Fermi gas model of one-dimensional conductors. Adv. Phys. 1979, 28, 201. [CrossRef]

18. Emery, V.J. Highly Conducting One-Dimensional Solids; Devreese, J.T., Evrard, R.E., van Doren, V.E., Eds.; Plenum: New York, NY, USA, 1979; p. 247.

19. Brazovskii, S.; Yakovenko, Y. On the theory of organic superconducting materials. Sov. Phys. JETP 1985, 62,1340 .

20. Caron, L.G.; Bourbonnais, C. Importance of one-dimensional correlations in the phase diagram of the $(\mathrm{TMTTF})_{2}$-(TMTSF $)_{2} \mathrm{X}$ salts. Phys. $B+C$ 1986, 143, 453. [CrossRef]

21. Bourbonnais, C.; Caron, L.G. New mechanisms of phase transition in quasi-one dimensional conductors. Europhys. Lett. 1988, 5, 209. [CrossRef]

22. Moret, R.; Pouget, J.P.; Comès, R.; Bechgaard, K. X-ray scattering evidence for anion ordering in the low-temperature phase of Di(Tetramethyltetraselanafulvalenium) Perrhenate. Phys. Rev. Lett. 1982, 49, 1008-1012. [CrossRef] 
23. Moret, R.; Pouget, J.P.; Comes, R.; Bechgaard, K. Structural phase transitions in (TMTSF) ${ }_{2} \mathrm{X}$ and (TMTTF) 2 X: A survey with some new results. J. Phys. Colloq. 1983, 44, C3-957. [CrossRef]

24. Foury-Leylekian, P.; Petit, S.; Andre, G.; Moradpour, A.; Pouget, J.P. Neutron scattering evidence for a lattice displacement at the charge ordering transition of (TMTTF) ${ }_{2} \mathrm{PF}_{6}$. Physica B 2010, 405, S95. [CrossRef]

25. Riera, J.; Poilblanc, D. Influence of anion potential on the charge ordering in the charge-transfer salts. Phys. Rev. B 2001, 63, R241102. [CrossRef]

26. Ilakovac, V.; Ravy, S.; Pouget, J.P.; Lenoir, C.; Boubekur, K.; Batail, P.; Babic, S.D.; Biskup, N.; Korin-Hamzic, B.; Tomic, S.; et al. Enhanced charge localization in the organic alloys [(TMTSF) $\left.{ }_{1-x} \mathrm{TMTTF}_{x}\right]_{2} \operatorname{ReO}_{4}$. Phys. Rev. $B$ 1994, 50, 7136. [CrossRef] [PubMed]

27. Penc, K.; Mila, F. Charge gap in the one-dimensional Hubbard model at quarter-filling. Phys. Rev. B 1994, 50, 11429. [CrossRef] [PubMed]

28. Tsuchiizu, M.; Yoshioka, H.; Suzumura, Y. Theoretical study of the charge gap of organic conductors Bechgaard salts. J. Phys. Soc. Jpn. 1999, 68, 1809. [CrossRef]

29. Monceau, P.; Nad, F.; Brazovskii, S. Ferroelectric Mott-Hubbard phase of organic (TMTTF) 2 X conductors. Phys. Rev. Lett. 2001, 86, 4080. [CrossRef] [PubMed]

30. Coulon, C.; Parkin, S.S.P.; Laversanne, R. Structureless transition and strong localization effects in bis-tetramethyltetrathiafulvalenium salts [(TMTTF) ${ }_{2}$ X]. Phys. Rev. B 1985, 31, 3583. [CrossRef] [PubMed]

31. Chow, D.S.; Zamborszky, F.; Alavi, B.; Tantillo, D.; Baur, A.; Merlic, C.A.; Brown, S.E. Charge Ordering in the TMTTF Family of Molecular Conductors. Phys. Rev. Lett. 2000, 85, 1698. [CrossRef] [PubMed]

32. Nad, F.; Monceau, P.; Carcel, C.; Fabre, J.M. Charge ordering phase transition in the quasi-one-dimensional conductor (TMTTF) ${ }_{2} \mathrm{AsF}_{6}$. J. Phys. Condens. Matter 2000, 12, L435. [CrossRef]

33. Seo, H.; Fukuyama, H. Antiferromagnetic phases of one-dimensional quarter-felled organic conductors. J. Phys. Soc. Jpn. 1997, 66, 1249. [CrossRef]

34. Mazumdar, S.; Clay, R.T.; Campbell, D.K. Bond-order and charge-density waves in the isotropic interacting two-dimensional quarter-filled band and the insulating state proximate to organic superconductivity. Phys. Rev. B 2000, 62, 13400. [CrossRef]

35. Riera, J.; Poilblanc, D. Coexistence of charge-density waves, bond-order waves, and spin-density waves in quasi-one-dimensional charge-transfer salts. Phys. Rev. B 2000, 62, R16243. [CrossRef]

36. Giamarchi, T. Mott transition in one dimension. Physica 1997, B230-232, 975. [CrossRef]

37. Pouget, J.; Moret, R.; Comes, R.; Bechgaard, K.; Fabre, J.M.; Giral, L. X-ray diffuse scattering of some $(\mathrm{TMTSF})_{2} \mathrm{X}$ and (TMTTF $)_{2} \mathrm{X}$ salts. Mol. Cryst. Liq. Cryst. 1982, 79, 129. [CrossRef]

38. Creuzet, F.; Bourbonnais, C.; Caron, L.G.; Jérome, D.; Bechgaard, K. A ${ }^{13} \mathrm{C}$ NMR study of the interplay between the spin-Peierls and antiferromagnetic ground states in (TMTTF) ${ }_{2} \mathrm{PF}_{6}$ under pressure. Synth. Met. 1987, 19, 289. [CrossRef]

39. Zamborszky, F.; Yu, W.; Raas, W.; Brown, S.E.; Alavi, B.; Merlic, C.A.; Baur, A. Competition and coexistence of bond and charge orders in (TMTTF) ${ }_{2} \mathrm{AsF}_{6}$. Phys. Rev. B 2002, 66, 081103(R). [CrossRef]

40. Sugiura, M.; Suzumura, Y. Competition of dimerization and charge ordering in the pin-Peierls state of organic conductors. J. Phys. Soc. Jpn. 2003, 72, 1458. [CrossRef]

41. Kuwabara, M.; Seo, H.; Ogata, M. Coexistence of charge order and spin-Peierls lattice distortion in one-dimensional organic conductors. J. Phys. Soc. Jpn. 2003, 72, 225. [CrossRef]

42. Tsuchiizu, M.; Yoshioka, H.; Suzumura, Y. Crossover from quarter-filling to half-filling in a one-dimensional electron system with a dimerized and quarter-filled band. J. Phys. Soc. Jpn. 2001, 70, 1460. [CrossRef]

43. Clay, R.T.; Mazumdar, S.; Campbell, D.K. Pattern of charge ordering in quasi-one-dimensional organic charge-transfer solids. Phys. Rev. B 2003, 67, 115121. [CrossRef]

44. Castet, F.; Fritsch, A.; Ducasse, L. Determination of coulombic interaction parameters of the extended Hubbard model in the organic conductors. J. Phys. I (France) 1996, 6, 583. [CrossRef]

45. Bychkov, Y.A.; Gorkov, L.P.; Dzyaloshinskii, I. Possibility of superconductivity type phenomena in a one-dimensional system. Sov. Phys. JETP 1966, 23, 489.

46. Bourbonnais, C.; Caron, L.G. Renormalization group approach to quasi-one-dimensional conductors. Int. J. Mod. Phys. B 1991, 05, 1033. [CrossRef]

47. Kimura, M. Possible phases and some properties of the one metal with the half-filled band. Prog. Theor. Phys. 1975, 63, 955. [CrossRef] 
48. Jacobsen, C.S.; Pedersen, H.J.; Mortensen, K.; Rindorf, G.; Thorup, N.; Torrance, J.B.; Bechgaard, K. An unusual metal-insulator transition: bis(tetramethyltetraselenafulvalenium)-perrhenate (TMTSF) ${ }_{2} \mathrm{ReO}_{4}$. J. Phys. C Solid State Phys. 1982, 15, 2651. [CrossRef]

49. Pustogow, A.; Petersein, T.; Kolatschek, S.; Engel, L.; Dressel, M. Electronic correlations versus lattice interactions: Interplay of charge and anion orders in (TMTTF) ${ }_{2}$ X. Phys. Rev. B 2016, 94, 195125. [CrossRef]

50. Liu, Q.; Ravy, S.; Senhaji, A.; Pouget, J.P.; Johanssen, I.; Bechgaard, K. Structural investigation of the $(\mathrm{TMDTDSF})_{2} \mathrm{X}$ series of organic conductors. Synth. Met. 1991, 42, 1711. [CrossRef]

51. Auban, P.; Jérome, D.; Johannsen, I.; Bechgaard, K. Physical properties of the new organic conductor series di(tetramethyl-dithiadiselenafulvalene) $\mathrm{X} ; \mathrm{X}=\mathrm{BF}_{4}, \mathrm{ClO}_{4}, \mathrm{ReO}_{4}, \mathrm{PF}_{6}, \mathrm{AsF}_{6}, \mathrm{SbF}_{6}$. Synth. Met. 1991, 41, 2285. [CrossRef]

52. Gotschy, B.; Auban-Senzier, P.; Farrall, A.; Bourbonnais, C.; Jerome, D.; Canadell, E.; Henriques, R.T.; Johanssen, I.; Bechgaard, K. One-dimensional physics in organic conductors (TMDTDSF) ${ }_{2} \mathrm{X}, \mathrm{X}=\mathrm{PF}_{6}$, $\mathrm{ReO}_{4}:{ }^{77} \mathrm{Se}-\mathrm{NMR}$ experiments. J. Phys. I (France) 1992, 2, 677. [CrossRef]

53. Grant, P.M. Electronic structure of the 2:1 charge transfer salts of tmtcf. J. Phys. Colloq. 1983, $44, \mathrm{C} 3847$. [CrossRef]

54. Jacko, A.C.; Feldner, H.; Rose, E.; Lissner, F.; Dressel, M.; Valenti, R.; Jeschke, H.O. Electronic properties of Fabre charge-transfer salts under various temperature and pressure conditions. Phys. Rev. B 2013, 87, 155139. [CrossRef]

55. Sato, N.; Saito, G.; Inokuchi, H. Ionization potentials and polarization energies of tetraselenafulvalene (TSF) derivatives determined from ultraviolet photoelectron spectroscopy. Chem. Phys. 1983, 76, 79. [CrossRef]

56. Parkin, S.S.P.; Mayerle, J.J.; Engler, E.M. Anion ordering in (TMTTF) ${ }_{2} \mathrm{ReO}_{4}$. J. Phys. (Paris) Coll. 1983, 44, C3-1105.

57. Coulon, C.; Delhaes, P.; Flandrois, S.; Lagnier, R.; Bonjour, E.; Fabre, J. A new survey of the physical properties of the (TMTTF) ${ }_{2} \mathrm{X}$ series. Role of the counterion ordering. J. Phys. (Paris) 1982, 43, 1059. [CrossRef]

58. Laversanne, R.; Coulon, C.; Gallois, B.; Pouget, J.P.; Moret, R. Structural and electrical properties of $(\mathrm{TMTTF})_{2} \mathrm{MF}_{6}$ salts $(\mathrm{M}=\mathrm{P}, \mathrm{As}, \mathrm{Sb})$. Role of the anions. J. Phys. (Paris) Lett. 1984, 45, L393. [CrossRef]

59. Su, W.P.; Schrieffer, J.R.; Heeger, A.J. Solitons in polyaecetylene. Phys. Rev. Lett. 1979, 42, 1698. [CrossRef]

60. Barišić, S.; Labbé, J.; Friedel, J. Tight-binding and transition-metal superconductivity. Phys. Rev. Lett. 1970, 25, 919. [CrossRef]

61. Caron, L.G.; Bourbonnais, C.; Creuzet, F.; Jerome, D. Evolution of the spin-Peierls transition in the (TMTTF $)_{2} \mathrm{X}$ salts $\left(\mathrm{X}=\mathrm{PF}_{6}, \mathrm{PF}_{6} \mathrm{Br}\right)$ under pressure. Synth. Met. 1987, 19, 69. [CrossRef]

62. Bourbonnais, C.; Dumoulin, B. Theory of lattice and electronic fluctuations in weakly localized spin-Peierls systems. J. Phys. I (France) 1996, 6, 1727. [CrossRef]

63. Scalapino, D.J.; Imry, Y.; Pincus, P. Generalized Ginzburg-Landau theory of pseudo-one dimensional systems. Phys. Rev. B 1975, 11, 2042. [CrossRef]

64. Ducasse, L.; Abderrabba, A.; Hoarau, J.; Pesquer, M.; Gallois, B.; Gaultier, J. Temperature dependence of the transfer integrals in the (TMTSF) ${ }_{2} \mathrm{X}$ and (TMTTF) ${ }_{2} \mathrm{X}$ families. J. Phys. C 1986, 19, 3805. [CrossRef]

65. Caron, L.G.; Creuzet, F.; Butaud, P.; Bourbonnais, C.; Jérome, D.; Bechgaard, K. Spin-Peierls and antiferromagnetic transition in $(\mathrm{TMTTF})_{2} \mathrm{PF}_{6}$ : EPR and NMR measurements and theory. Synth. Met. 1988, 27B, 123. [CrossRef]

66. Chow, D.; Wzietek, P.; Foglatti, D.; Alavi, B.; Tantillo, D.J.; Merlic, C.A.; Brown, S.E. Singular behavior in the pressure-tuned competition between spin-Peierls and antiferromagnetic ground states of $(\mathrm{TMTTF})_{2} \mathrm{PF}_{6}$. Phys. Rev. Lett. 1998, 81, 3984. [CrossRef]

67. Yu, W.; Zhang, F.; Zamborszky, F.; Alavi, B.; Baur, A.; Merlic, C.A.; Brown, S.E. Electron-lattice coupling and broken symmetries of the molecular salt ?(TMTTF) ${ }_{2} \mathrm{SbF}_{6}$. Phys. Rev. B 2004, 70, R121101. [CrossRef]

68. De Souza, M.; Squillante, L.; Sônego, C.; Menegasso, P.; Foury-Leylekian, P.; Pouget, J.P. Probing the ionic dielectric constant contribution in the ferroelectric phase of the Fabre salts. Phys. Rev. B 2018, 97, 045122. [CrossRef] 
69. De Souza, M.; Pouget, J.P. Charge-ordering transition in (TMTTF) 2 X explored via dilatometry. J. Phys. Condens. Matter 2013, 25, 343201. [CrossRef] [PubMed]

Publisher's Note: MDPI stays neutral with regard to jurisdictional claims in published maps and institutional affiliations.

(C) 2020 by the authors. Licensee MDPI, Basel, Switzerland. This article is an open access article distributed under the terms and conditions of the Creative Commons Attribution (CC BY) license (http://creativecommons.org/licenses/by/4.0/). 